\title{
Villes et agglomérations suisses
}

\section{Le modèle d'évolution urbaine helvétique: une centralité qui se métamorphose}

L'évolution du système territorial helvétique, et singulièrement celle de son système urbain, ont maintenant reçu toute l'attention qu'elles méritaient, leurs principales phases ont été identifiées et interprétées, voire réinterprétées dans leurs invariances comme dans leurs changements, tant formels que structurels, de manière généralement jugée satisfaisante par le consensus des chercheurs (ROSSI 1983, CUNHA et RACINE 1992, CUNHA 1995, CUNHA et RACINE 1995), l'accent ayant été mis par ces derniers sur le phénomène clé de métropolisation (BAS. SAND, JOYE et SCHULER 1988, LERESCHE, JOYE, BASSAND 1995) dont on découvre progressivement les liens avec la globalisation de l'économie mondiale (CUNHA 1995).

Depuis 1970 , le système territorial suisse est entré dans une phase de transition urbaine caractérisée par une décroissance démographique des centres des agglomérations qui se réalise principalement au profit d'une forte croissance des communes suburbaines et périurbaines. Entre 1970 et 1990, la croissance démographique des grandes agglomérations reste positive, encore qu'elle soit essentiellement portée par l'augmentation des populations de leurs couronnes. Par ailleurs, si les petites et moyennes agglomérations poussent très fort (FREY 1996), force est de réaliser que la croissance de nombreux petits centres est le plus souvent déterminée par l'extension et le rayonnement des grands centres urbains (SCHULER 1994).

Corrélativement, la mobilité croissante des résidents entre des espaces dont les affectations tendent à se spécialiser est devenue l'un des phénomènes premiers de la géographie helvétique. Entre 1950 et 1990, dans les cinq grandes agglomérations suisses, le nombre de personnes sortant quotidiennement de leur commune de domicile pour se rendre au travail a augmenté de six fois pour dépasser, au total, 600000 navetteurs. A. DOzIO (1995) a récemment montré que la propagation spectaculaire de la mobilité quotidienne est intimement liée à la dispersion de la population urbaine dans les grandes régions métropolitaines et aux mutations de l'organisation économique de la production et de l'échange.

Les résultats sont nets: les activités tertiaires sont davantage concentrées dans les zones urbaines par rapport aux activités industrielles et à la population; les services aux entreprises et les services de distribution (étroitement corrélés dans l'espace suisse) sont plus «urbains» et étroitement liés à l'emploi qualifié (les premiers dans l'hyper- centre, les seconds plutôt liés aux nouveaux centres secondaires et aux couronnes des agglomérations) que les services sociaux et les services personnels qui accompagnent fidèlement les mouvements de déconcentration résidentielle périphérique et l'étalement rampant du champ de consommation des citadins.

Autant de résultats qui recoupent en général ce qui se passe un peu partout aujourd'hui dans le monde développé occidental et qui renvoient plus aux théories de la division territoriale du travail (AYDALOT 1985) qu'à celle des lieux centraux. Non pas tant que la hiérarchie des lieux centraux soit remise en cause, mais parce qu'à l'évidence, la centralité urbaine se métamorphose, n'étant plus seulement liée à l'exercice des services aux résidents, mais aussi à l'aptitude de chaque ville à reproduire une matrice de qualifications spécifiques, corrélée à des «complexes d'activités tertiaires» plus ou moins spécialisées destinées autant aux ménages qu'aux entreprises. L'hypothèse a été vérifiée: le système hiérarchisé des lieux centraux structure la qualité des flux migratoires. L'énoncé «à ville plus grande, travail plus qualifié» se double d'un second: «à formation plus élevée, mobilité plus forte vers les plus grandes agglomérations". A ceci près que l'on observe également des différences significatives entre niveaux hiérarchiques et mobilité des individus ayant une formation moins élevée (CUNHA et RACINE 1996).

De fait, l'analyse comparative de P. DANIELS (1993) le confirme: la diversité des ressources humaines, leur niveau de formation, leur compatibilité linguistique, leur mobilité, leur familiarité avec les pratiques d'affaires nationales et internationales, etc. sont des atouts majeurs pour les grandes agglomérations urbaines. Un constat qui permet de mieux spécifier la notion de centralité urbaine, montrant bien que le pouvoir décisionnel des villes continue à s'étendre sur l'ensemble du territoire national malgré le ralentissement démographique de leurs villes-centre. C'est ce que traduit également l'évolution de l'inscription au sol des niveaux de formation et des statuts socio-professionnels depuis 1970 (CUNHA 1995).

Le résultat de l'ensemble des travaux conduits en Suisse le confirment également: le gradient des niveaux de formation et de qualification selon la taille des villes et

Pierre Dessemontet, Ass. dipl., Jean-Bernard Racine, Prof., Université de Lausanne, Institut de Géographie BFSH-2, 1015 Lausanne 
agglomérations semble bien être favorable au redéploiement des grandes agglomérations et de leurs aires métropolitaines. Mais si, autre résultat très général dans nos pays, en captant de fait, à l'heure de la globalisation, les principaux dynamismes économiques, la grande ville reste le grand multiplicateur d'alternatives économiques et sociales et le lieu privilégié de communication et d'interaction sociale, et donc le moteur des restructurations économiques en cours, elle focalise aussi les problèmes de précarité économique, de pauvreté et d'exclusion sociale qui lui sont liés.

La globalisation est un processus économique fondé sur le décloisonnement des espaces, la multiplication des échanges extérieurs, l'interdépendance des marchés, mais aussi sur la rationalisation des moyens et des ressources d'entreprises de plus en plus soumises à une idéologie productiviste qui accentue la précarité et les inégalités sociales. Globalisation, métropolisation, tertiairisation, qualification sélective et dynamisme économique, certes, mais aussi, métropolisation, précarisation de l'emploi et exclusion sociale. La géographie sociale des aires métropolitaines helvétiques confirme la prégnance de tous ces phénomènes tout en montrant qu'ils obéissent pour partie à certains biais régionaux.

Force est de constater cependant que le modèle de la croissance urbaine alimentée par l'exode rural ayant vé$\mathrm{cu}$, le principal vecteur de cette croissance d'ensemble est lié à l'apport des migrations internationales. La tertiarisation et l'affinage des grands centres urbains attirent vers eux la main-d'œuvre la plus qualifiée, le marché du travail urbain en matière de main-d'œuvre peu qualifiée se faisant également au profit des plus grands centres. Par ailleurs, la périurbanisation des classes dirigeantes d'origine suisse bénéficie aux couronnes relativement lointaines, tandis que la déconcentration relative des activités industrielles vers les villes et les communes de petite dimension délocalise une partie relativement plus importante des formations professionnelles dont la "distance» à l'emploi industriel est systématiquement plus faible. En d'autres termes, l'évolution tant spatiale que sociale de ces vingt dernières années a sans doute reconfiguré, dans le cadre du phénomène de métropolisation, la géographie sociale de nos agglomérations.

A cet égard un certain nombre d'hypothèses pouvaient être formulées en regard de ce qui différenciait entre elles à l'échelle globale, les 48 agglomérations suisses et 9 villes isolées définies par l'OFS (SCHULER 1994) en 1990, à l'échelle plus locale, les 805 communes concernées.

Hypothèse 1: Le rôle du facteur taille. Les différences entre les agglomérations et entre les communes d'une agglomération sont-elles tributaires du facteur taille? La réponse attendue est positive: plus une agglomération est grande, plus la probabilité est forte d'y voir apparaître certains phénomènes spécifiques, qu'il s'agisse de types de formations, de structures socio-professionnelles, de biens, de services, de phénomènes culturels ou sociaux. Par ailleurs, plus une agglomération est grande, plus les communes qui la composent devraient être différenciées entre elles.
Hypothèse 2: Le rôle du facteur régional. Indépendamment de l'effet de taille, si celui-ci se confirme, les différences entre les agglomérations sont-elles plus ou moins significatives que les différences internes, le facteur régional se révélant alors plus important que les ségrégations internes? La question est de savoir, en d'autres termes, et par exemple, si les différences entre communes riches et pauvres zurichoises sont moins importantes que les différences entre communes riches zurichoises et communes riches lausannoises. Ce qui confirmerait, entre autres, l'existence postulée par J. L. PIVETEAU (1982) de deux sous-systèmes urbains contrastés de part et d'autre de la Sarine.

Hypothèse 3: La métropolisation. Elle renvoie au rôle particulier de l'émergence du phénomène métropolitain dans quelques centres privilégiés avec leurs couronnes spécifiques. Qu'est-ce qui différencie, dans la dispersion des attributs sociaux communaux les villes relevant du niveau métropolitain? Si spécificités il y a, celles-ci sontelles réservées aux villes-centre ou bien s'en dégagentelles? Si oui, pour lesquelles?

Il n'est donc pas indifférent aujourd'hui d'essayer de radiographier à nouveau les grandes articulations structurelles du système urbain helvétique en dégageant les principaux facteurs de la différenciation socio-spatiale des communes d'agglomération d'une part, les rapports qu'ils entretiennent avec les structures hiérarchiques du système urbain et les éventuelles variations régionales qui se dégagent de la manière dont ils s'inscrivent dans le territoire helvétique d'autre part, les types de combinaisons enfin qui en dérivent et contribuent à définir la Suisse urbaine d'aujourd'hui. Accessoirement, nous saisirons l'occasion pour découvrir l'existence éventuelle de certaines liaisons entre ces structures socio-spatiales et certains types de comportements politiques en particulier.

\section{Les principaux facteurs de la différenciation socio- spatiale des agglomérations suisses en 1990}

On doit à M. SCHULER (1994) d'avoir revu, pour le compte de l'Office fédéral de la statistique, la définition des agglomérations suisses. Complexe, extensive et très fonctionnaliste, la nouvelle définition favorise évidemment l'image toujours plus diffuse de l'urbanisation helvétique et l'inévitable interpénétration des régions urbaines en même temps qu'elle souligne le renforcement de la hiérarchie entre les agglomérations.

C'est dans cet ensemble ainsi découpé que nous avons associé à chacune des 805 communes concernées une série d'observables considérées comme autant d'attributs socio-économiques (répartition des emplois dans les grands secteurs, niveaux de formation et revenus, classes socio-professionnelles) et subsidiairement démographiques (densité, rapport de dépendance et rapport de renouvellement), saisis pour les uns au lieu de résidence, pour d'autres, et parfois les mêmes, au lieu de tra- 
vail, en ce qui concerne la répartition des grands secteurs économiques, les classes socio-professionnelles, certaines typologies d'emplois, la participation enfin des femmes à la population active.

On notera enfin que devant être soumis à l'hypothèse factorielle de la possibilité de réduire cette information considérable à quelques composantes principales sur la base des colinéarités existant au sein de la matrice d'information, ces variables ont d'abord été filtrées pour éliminer les redondances de contenu et ne garder, sauf exception justifiable, que les descripteurs entretenant avec l'un d'entre les autres au moins une corrélation supérieure à 0,7 .

De fait, on l'a vu, l'émergence du phénomène métropolitain est liée au rôle clé de quelques centres privilégiés avec leurs couronnes spécifiques. L'analyse en composantes principales révèle bien l'existence d'articulations sous-jacentes, généralement reconnues ailleurs dans le monde occidental, mais donnant aux formes socio-spatiales de l'urbanisation métropolitaine helvétique une forte connotation régionale.

\section{La Suisse des banlieues riches}

Une première composante, obtenant le poids maximal en termes de variance expliquée $(22,1 \%$, tableau 1$)$ renvoie, très spécifiquement, aux regroupements et oppositions socio-économiques parmi les habitants des communes. Il s'agit d'un facteur identifiant les communes refuge des classes dominantes, combinaison de variables mesurant la part des plus hauts revenus, des universitaires, le poids des professions intellectuelles et d'encadrement, professions directoriales et libérales des couronnes aisées des grands centres, avec une forte spécificité de la région genevoise et de la Rive dorée zurichoise, par opposition à l'importance des professions ouvrières et des plus bas revenus des périphéries des petites agglomérations valaisannes, grisonnes, bernoises. Il s'agit à l'évidence d'une véritable "classe socio-spatiale», se définissant à l'échelle régionale et locale. On remarquera que sur la hiérarchie des scores, les centres des cinq plus grandes agglomérations sont neutres sauf en ce qui concerne celui de $\mathrm{Ge}$ nève $(0,83$, qui représente une exception). En revanche ce sont bien des communes périurbaines liées aux "cinq grandes» qui sont mises en évidence. La première commune qui n'est pas liee à l'une des cinq n'arrive en effet qu'en 55 e position.

Le cas le plus spectaculaire reste celui de Genève (carte 1). L'essentiel de l'agglomération genevoise est en effet marqué très positivement sur ce facteur, le centre comme la quasi-totalité des communes de l'agglomération, ce qui est un cas unique en Suisse. Cette apparente globalité de la richesse genevoise est probablement due à un taux d'universitaires autrement plus élevé qu'en moyenne helvétique, ainsi qu'à la présence à Genève même des institutions internationales et de très nombreux centres sociaux nécessitant une main-d'œuvre très qualifiée. $\mathrm{Au}$ sein de l'agglomération, certaines communes ressortent, notamment rive droite du lac, et en Terre-Sainte vaudoise, deux régions qui abritent les communes les plus marquées de Suisse sur cet axe.

Les autres grandes agglomérations répondent mieux au modèle que nous avons esquissé plus haut. C'est assez logiquement à Zurich qu'il est le plus marqué, avec les communes de la Goldküste du lac de Zurich. La région située en face de la Goldküste ressort également, ainsi que certaines communes de l'Albis (Uitikon en tête): il se définit ainsi une sorte de ceinture sud autour de Zurich et de son lac qui regroupe l'essentiel des belles banlieues zurichoises. Lausanne a aussi ses belles banlieues: deux îlots à l'ouest, l'ensemble de l'est de l'agglomération, mais également, de plus en plus, dans les banlieues lointaines de la Côte, ainsi que dans le nord. Le phénomène est nettement moins marqué à Bâle et à Berne, tout en restant notable.

Dans le reste de la Suisse urbaine, le phénomène est beaucoup moins présent, sauf en Suisse latine, où il se lit très bien encore au Tessin, autour de Lugano, mais aussi dans la plupart des agglomérations moyennes romandes, notamment dans celle de Vevey-Montreux (il se définit ainsi un arc lémanique qui apparaît favorisé), dans celles de Fribourg et de Neuchâtel. En Suisse alémanique et dans l'essentiel de l'arc alpin, le phénomène de l'individualisation des communes riches est quasiment absent, très faible dans des agglomérations de bonne taille comme Lucerne, Winterthour ou St-Gall, totalement absent ailleurs, à l'exception de Baden (encore faudrait-il pouvoir distinguer si la ségrégation à l'intérieur de l'agglomération de Baden est indépendante de l'influence zurichoise).

Il apparaît ainsi que la différenciation entre classes dominantes et autres classes est plus nette dans les grandes agglomérations, mais également, de manière générale, dans l'ouest du pays (Moyen-Pays romand notamment) alors qu'il semble que les classes sociales soient, à taille d'agglomération égale (le phénomène zurichois et de ses rives dorées doit être mis à part), mieux mélangées en Suisse alémanique qu'en Suisse romande, signe peut-être d'une plus grande unité et d'une meilleure cohésion sociale.

Les classes dirigeantes du pays se concentrent donc dans le Moyen-Pays, les Alpes étant de ce point de vue nettement à la traîne, ce qui implique un déficit structurel, leur évidente fragilité face au reste du pays. D'autre part, certaines régions du Moyen-Pays en sont également dépourvues: c'est le cas dans la majorité des petites agglomérations, en Suisse alémanique surtout, notamment dans l'est industriel du pays et dans le grand tentacule argovien issu de l'agglomération zurichoise, qui s'étire de Baden à Olten.

\section{Centres d'emplois urbains versus périphéries "rurbaines": une hiérarchie indiscutable}

Une deuxième composante (réunissant $13,6 \%$ de la variance totale, tableau 2) indexe manifestement la centrali- 
té différentielle des communes, expression combinée de l'importance des employés sur leur lieu de travail, des communes où l'emploi l'emporte sur la fonction résidentielle, de la densité, des étrangers et des actifs féminins domiciliés sur place. S'y associent aussi bien des professions intellectuelles et d'encadrement au lieu de travail que des employés et des non-qualifiés au domicile. Les agglomérations sont toutes au sommet de la hiérarchie des scores, mais leur première couronne les y accompagnent. La ville de Genève obtient le score maximum suisse, à 2,94 écarts-type de la moyenne, mais la centralité aujourd'hui déborde les limites des villes-centre sur quelques communes arrivées à un certain degré de maturité (communes matures des anglo-saxons), qualifiant ainsi le niveau atteint par la ville-centre dans la hiérarchie urbaine, mais exprimant spatialement l'émergence de corridors en gestation et la fin annoncée du modèle mono-centré, en voie de métamorphose vers une centralité réticulée.

Le pôle négatif décrit l'importance résiduelle de l'agriculture au sein même des limites des agglomérations définies par l'OFS. Le rapport démographique de dépendance s'y associe, ce qui tendrait à montrer que de manière générale les campagnes vieillissent. Se distinguent du côté négatif de ce second facteur les communes où sur un substrat rural qui subsiste est venu s'implanter une population «rurbaine». Tout concourt ici à définir ce facteur comme étant le facteur centre-périphérie, et phénomène remarquable, bien défini au sein même des agglomérations.

Lobservation de la carte (carte 2) montre qu'il n'existe pas de variation régionale majeure entre les différentes agglomérations du pays. Toutes comportent un centre assez aisément identifiable; dans leur quasi-totalité, ces centres ne recouvrent pas la majeure partie de l'agglomération. La structure interne des agglomérations est partout ou presque la même, clairement concentrique. En bref, une carte attendue, clairement prévisible. Elle a cependant une utilité évidente. Outre le fait de délimiter clairement les centres, elle permet notamment de mesurer à quel point les "centres vrais» des agglomérations dépassent les limites communales des villes titulaires.

La zone centrale de l'agglomération de Zurich, centre économique majeur du pays, s'étend comme nulle part ailleurs dans le pays, non pas en annexant une ou deux communes limitrophes, mais en étendant ses tentacules loin à la ronde. Outre Zurich font partie intégrante du centre de l'agglomération la quasi-totalité des communes limitrophes, notamment dans le nord de la ville. Zurich déploie en outre trois tentacules clairement urbains: un en direction de Baden, long de près de $20 \mathrm{~km}$; un second, sur la rive sud-ouest du lac de Zurich, se déploie jusqu'à Thalwil en direction de Zoug; un troisième, à l'est, rejoint la commune d'Uster. Au-delà et autour de ces tentacules, la grande majorité des communes de l'agglomération zurichoise sont clairement urbaines, ce qui est un phénomène unique en Suisse.

Les autres grandes agglomérations de Suisse comportent des centres nettement plus petits, qui n'ont pas (encore?) généré ces tentacules éminemment urbains présents à Zurich. Seconde ville du pays pendant longtemps, Bâle n'a clairement annexé que la commune de Birsfelden, quand bien même de Bâle à Liestal et à Rheinfelden, la chimie a établi un corridor urbain qui laisse penser à la formation d'un tentacule, bien moindre cependant que ses homologues zurichois. Le centre se développe également vers le sud. Mais au contraire de Zurich, une grande partie de la périphérie bâloise reste encore fortement rurale. Berne ne sort pratiquement pas de ses limites. On passe très vite en effet du centre-ville aux banlieues encore fortement marquées par l'agriculture. On peut plus facilement adjoindre à Lausanne la commune de Pully, mais surtout celles de l'ouest industriel. Malgré cette générosité, le centre de l'agglomération apparaît peu étendu en regard de ceux de Bâle et surtout de Zurich, et ses périphéries n'échappent pas à une forte présence du secteur primaire. Si cette dernière remarque vaut également pour l'agglomération genevoise, le centre de cette dernière est plus étendu, et surtout plus net qu'à Lausanne, concernant un axe s'étendant de Meyrin aux deux Chêne et regroupant une dizaine de communes. Cet axe n'est pourtant pas comparable aux débordements zurichois. Plus les agglomérations sont modestes, plus leur centre se réduit à la commune titulaire. Lugano, qui déborde sur quelques minuscules communes alentour, fait exception à cette règle. Mais tant Lucerne que Winterthour et St-Gall ne connaissent pas de débordements extra-muros. Cette situation est générale dans les petites agglomérations, dont la quasi-totalité des communes rattachées sont encore fortement agricoles, à la majeure exception de la grande excroissance argovienne qui semble être constituée d'un couloir urbain sans réelle rupture et qui prolonge jusqu'à Olten le tentacule Zurich-Baden. A l'exception également de certaines agglomérations moyennes comme Fribourg ou encore Neuchâtel, Bellinzone, Locarno.

En résumé, on constate qu'existent en Suisse quatre classes hiérarchiques de centres d'agglomération. Zurich constitue l'unique cas de la classe supérieure, avec un centre qui non seulement déborde très largement de la commune, en recouvrant la totalité des communes adjacentes, mais qui se prolonge également en tentacules urbains qui rayonnent du centre et qui se mesurent en dizaines de kilomètres: à l'aune de ce facteur qui se présente comme un véritable indice d'urbanité des communes, Zurich comporte un centre tentaculaire urbain unique en Suisse. Plus largement, on peut reellement parler d'une aire métropolitaine compacte qui s'étend d'Olten à Frauenfeld, de Schaffhouse à Zoug et Lachen, entre lesquels la continuité urbaine est manifeste, évidente: un phénomène totalement inconnu ailleurs, «métropole lèmanique» comprise.

Deuxième classe, celle des centres classiques de grandes agglomérations. C'est celle de Genève et Bâle surtout, mais aussi de Berne et Lausanne. Aires centrales fortes qui débordent largement de la commune centrale, embryons de tentacules (à Bâle et Genève), mais globalement, si le centre est étendu, il reste entouré de banlieues 
encore moyennement urbanisées, au paysage où l'élément agricole joue encore un rôle certain.

Troisième classe, celle des villes moyennes: un centre encore essentiellement ponctuel, bien moins étendu que ceux de la classe précédente, mais qui souvent a commencé à déborder sur les communes voisines, sans pour autant que cela ait altéré leur forme circulaire et la nette tendance "rurale» de leurs couronnes. Le quatrième groupe se compose enfin des petites agglomérations (de 20000 à 40000 habitants), dont seule la commune centrale fait preuve d'une certaine urbanisation et dont le cas le plus pur est celui des villes isolées qui n'ont pas encore rattaché des communes externes pour former une agglomération.

\section{Triangle d'Or versus Suisse latine, ou la Suisse des "arts et métiers" contre la Suisse des "petites mains"}

Le troisième facteur (tableau 3 ) discrimine de nouveau et avant tout des lieux de résidence. Majeure par ses saturations, une première opposition indexe les niveaux de formation: le pôle positif montre une surreprésentation des personnes étant au bénéfice d'une formation professionnelle achevée, alors que le pôle négatif montre une surreprésentation des personnes n'ayant suivi qu'une formation scolaire obligatoire. En d'autres termes, un gradient de formation qui ne touche pas les formations supérieures, mais bien les qualifications moyennes, entre une Suisse fortement dotée en employés et ouvriers qualifiés et une Suisse où les personnes sans formation dominent.

A cette première opposition se superpose une seconde opposition, dans les types de profession cette fois: les professions intermédiaires, en positif, sopposent aux non-qualifiés en négatif, seconde opposition assez logique en regard de la première à laquelle elle est nettement liée. Combinées, elles définissent la structure fondamentale du facteur, la Suisse du travail qualifié, du «bon métier», du pari sur la qualité du travail, versus la Suisse des masses de travailleurs non qualifiés, des manœuvres. Cette opposition entre la Suisse «des arts et métiers» et celle des manœuvres recouvre également, quoique de manière plus diffuse, une opposition dans les revenus, puisque les revenus moyens à aisés sont surreprésentés sur le pôle positif, alors que les faibles revenus le sont sur le pôle négatif. Les autres variables ne jouent pas de rôle réellement significatif sur la formation du facteur, si ce n'est que la proportion d'ètrangers est plus forte du côté négatif du facteur.

En d'autres termes, une opposition assez cohérente entre deux types bien déterminés de main-d'œuvre (par opposition à "classe dirigeante»): une main-d'œuvre bien formée, exerçant des professions qualifiées rétribuées correctement s'oppose à une main-d'œuvre non formée, exerçant des emplois non qualifiés mal rétribués, phénomène souligné par la présence d'une forte main-d'œuvre immigrée: la dichotomie classique entre classes moyennes et «servantes» de la société.
Il est dès lors du plus haut intérêt de comparer cette dimension structurelle au premier facteur de l'analyse, qui portait également sur des variables de formation, de qualification professionnelle et de revenus. Le premier facteur distinguait clairement les classes dominantes, très bien formées, exerçant des responsabilités et gagnant très bien sa vie, du reste de la population; le troisième facteur s'intéresse plus clairement à ce "reste de la population", qu'il sépare en deux groupes selon leur type de formation, de professions et de revenus. L'individu statistique étant ici "territorial», c'est dire que dans le territoire suisse, l'habitat urbain se discrimine fortement en différentes strates sociales, entre communes qui hébergent les classes dominantes, celles qui sont le bastion d'une classe moyenne qui a fait ce pays, celles enfin qui sont avant tout des réservoirs de main-d'œuvre bon marché.

Spatialement (carte 3), le Triangle d'Or constitue le principal bloc des agglomérations à main-d'œuvre qualifiée, un triangle dont les angles évidents sont Zurich, Bâle et Berne. Au sein de cet ensemble, les communes à forte proportion de main-d'œuvre qualifiée dominent largement le paysage, et ce dans la quasi-totalité des agglomérations concernées. A première vue, la Suisse urbaine prise dans son ensemble a donc un cœur de main-d'œuvre qualifiée, un cœur entièrement alémanique et largement protestant, recouvrant une aire géographique limitée à l'ouest par la limite linguistique, au sud par les Préalpes, à l'est par une ligne Schaffhouse-Winterthour-Rapperswil, au nord par le Rhin: le cœur de la Suisse alémanique.

Au sein de ce grand ensemble, des dichotomies apparaissent néanmoins selon une double échelle, régionale et locale: les agglomérations de ce grand bloc se différencient entre elles comme elles se différencient en leur sein. Au plan régional, la proportion de main-d'œuvre qualifiée est en règle générale plus forte dans les grandes agglomérations qu'ailleurs, dans l'agglomération zurichoise en premier lieu. Celle-ci "écrase» le bloc et la Suisse de ce point de vue, et s'adjoint la majorité des agglomérations limitrophes. Derrière ce "heartland" primordial, les deux autres grandes agglomérations de Bâle et de Berne, auxquelles Lucerne s'associe d'ailleurs, ressortent également fortement, plus fortement en tous cas que les autres agglomérations du bloc, un peu à la traîne (toutes proportions gardées), surtout à l'ouest (Zurich influençant l'est). On constate par exemple que la partie ouest du tentacule argovien est moins profilé que le reste du bloc, ce qui est également le cas de la majorité des petites agglomérations du bloc. A l'extérieur de ce bloc quasiment uni, qui ne compte pas d'exception marquante, peu d'agglomérations montrent une forte proportion de main-d'œuvre qualifiée. C'est pourtant le cas dans les Grisons, dont les deux centres urbains (Coire et Davos) sont nettement profilés du côté positif du facteur. Le reste de la Suisse alémanique, à savoir le petit axe horloger de Bienne à Soleure, voire Olten, la Suisse centrale et surtout orientale à tradition textile, tendent à se profiler plutôt du côté négatif de l'axe, ce qui dénote sans doute la fragilité de la maind'œuvre dans ces régions spécialisées dans des types 


\section{Géographie de la force de travail de la Suisse urbaine, 1990}

(805 unités spatiales et 46 caractéristiques)

Analyse en composantes principales, solution Varimax à 5 composantes, $62.9 \%$

\section{Composante 1}

Variance expliquée : $22.1 \%$

Variance cumulée : $\quad 22.1 \%$

\section{Structure}

Caractéristiques

Saturations +

Profil corrélatif des variables sur l'axe

$\begin{array}{llll}\text { Revenus supérieurs à } 75 \mathrm{kF} & 0.95 & \text { Form. professionnelle } & -0.37 \\ \text { Universitaires } & 0.90 & \text { Ouvriers au travail } & -0.39 \\ \text { Prof. intellectuelles et d'enc.(dom)0.89 } & & \text { Rapport de renouvellement } & -0.46 \\ \text { Titulaires de maturité } & 0.78 & \text { Form. scolaire obligatoire } & -0.56 \\ \text { Prof. directoriales au dom. } & 0.75 & \text { Travailleurs non qual. au dom. } & -0.58 \\ \text { Prof. libérales au dom. } & 0.72 & \text { Revenus inférieurs à 20 kF } & -0.60 \\ \text { Form. prof. supérieures } & 0.69 & \text { Travailleurs secondaires au dom. } & -0.61 \\ \text { Travailleurs tertiaires au dom. } & 0.58 & \text { Revenus entre } 40 \text { et } 50 \mathrm{kF} & -0.67 \\ \text { Prof. libérales au trav. } & 0.54 & \text { Revenus entre 20 et } 30 \mathrm{kF} & -0.72 \\ & & \text { Revenus entre 30 et } 40 \mathrm{kF} & -0.73 \\ & & \text { Ouvriers au dom. } & -0.84\end{array}$

Saturations -

Poids locaux

Projection des unités spatiales sur l'axe

Unités spatiales Ecarts-types

1. Cologny (Genève)

2. Bogis-Bossey (Genève)

3. Commugny (Genève)

4. Dully (Genève)

5. Chavannes-de-Bogis (Genève) 3.20

15. Jouxtens-Mézery (Lausanne) 2.62

18. Uitikon (Zürich) 2.57

55. Cheseaux-Noréaz (Yverdon) 1.73

59. Biel-Benken (Basel) 1.70

81. Bolligen (Bern) $\quad 1.45$

147. Genève

0.83
336. Zürich

$-0.02$

357. Lausanne

$-0.07$

416. Bern

$-0.22$

426. Basel

$-0.24$

470. St-Cergue (Genève) $\quad-0.34$

548. Carrouge (Lausanne) $\quad-0.56$

694. Höri (Zürich) -0.95

696. Grellingen (Basel) $\quad-0.95$

729. Altstätten -1.07

751. Wünnewil-Flamatt (Bern) $\quad-1.17$

801. Bönigen (Interlaken) $\quad-1.78$

802. Bitsch (Brig-Visp) -1.89

803. Mastrils (Chur) -1.89

804. Termen (Brig-Visp) $\quad-2.01$

805. Eggerberg (Brig-Visp) $\quad-2.81$

\section{Définition de la composante}

Classes dominantes versus ouvriers, faibles revenus et faibles formations au lieu de résidence ou la Suisse des banlieues riches 


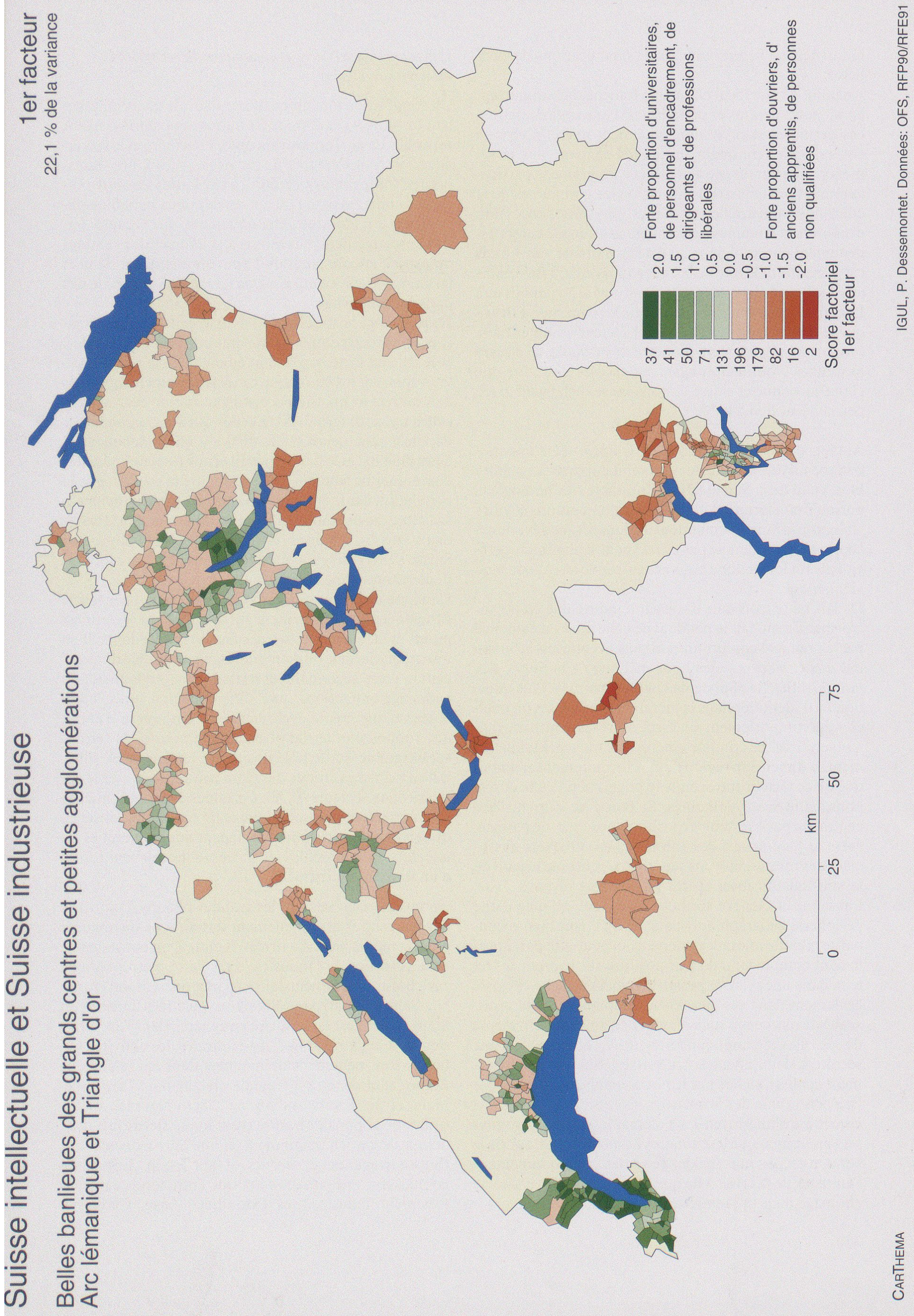


industriels ne nécessitant pas de qualifications particulières.

Au point de vue local enfin, une franche dichotomie apparaît entre centres et périphéries d'une agglomération, ces dernières étant systématiquement mieux pourvues en main-d'œuvre qualifiee que les centres, plus largement habité par les «non-qualifiés». Phénomène particulièrement bien illustré à Zurich, où les communes de la couronne industrielle ont toutes une forte proportion d'ouvriers et d'employés qualifiés, alors que le centre est nettement moins bien pourvu de ce point de vue. Le cas est général. On le retrouve, encore plus aigu, à Bâle, mais aussi à Berne, dans les agglomérations argoviennes et à Lucerne. On notera par ailleurs que les communes riches qui se détachaient sur le premier facteur comptent une proportion relativement modeste, voire faible, de classes moyennes (voir la Goldküste zurichoise): c'est dire que classe moyenne et classe dominante se mélangent assez peu sur leur lieu d'habitat.

Autant, dans son ensemble, le Triangle d'Or apparaît comme un tout homogène, autant il s'oppose à un autre bloc, celui de la Suisse latine. Ensemble très homogène, puisqu'il concerne la totalité des agglomérations et villes isolées de la Suisse romande et du Tessin, sans aucune exception. On n'y dénote pas en revanche les hiérarchies régionales et locales que nous avons trouvé au sein du bloc alémanique.

De fait, cette troisième dimension indexe une classe socio-spatiale qui fait le pendant de celle mise en évidence par la première composante, opposant cette fois la Suisse des arts et métiers ("des bons métiers») à la Suisse des non-qualifiés (la "Suisse des petites mains»). Plus question ici d'identifier dans la hiérarchie des scores un quelconque comportement analogue entre les centres des agglomérations, ce comportement fut-il moyen. Manifestement, la dimension régionale et prioritairement alémanique de ce facteur transcende le phénomène taille et responsabilité métropolitaines. Si Berne et Zurich, l'axe central du pays, restent en position moyenne, de partage, entre le pôle positif des "professions" auxquels s'associent des revenus moyens supérieurs, et le pôle négatif de la sous-qualification et des plus faibles revenus, Bâle, Lausanne et Genève surtout s'inscrivent, au-delà d'une périphérie géographique, dans ce qu'il faut bien reconnaître comme relevant également, et peut-être paradoxalement compte tenu de leur position sur le premier facteur, dans une relative périphérie sociale. Celle-ci existe également dans ces centres-là: témoignage de la croissance de morceaux socialement déprimés au sein même de ces villes et expression fort nette de l'émergence d'une société à deux vitesses. La Suisse latine est manifestement la plus touchée par le phénomène auquel s'oppose ce glacis urbain des formations professionnelles des arts et métiers d'un Moyen-Pays alémanique chérissant plus ses «apprentis» que les titulaires de maturité. Ce qui, on le verra, n'est pas sans incidence sur les comportements et l'ampleur de la crise, telle que mesurée par l'intensité du chômage dans la première moitié de la décennie.

\section{Communes du tertiaire et communes du secondaire: un territoire bigarré}

Une opposition fondamentale structure le quatrième facteur (tableau 4) et domine les autres oppositions, si tant est qu'il y en ait: l'opposition entre le tertiaire et le secondaire, entre les centres de services et les villes industrielles. Cette opposition attire à elle toutes les autres variables concernées, à l'exception des deux variables «féminines» qui montrent, classiquement, que l'emploi tertiaire est largement plus ouvert aux femmes que l'emploi industriel; encore faudrait-il pouvoir distinguer de quel tertiaire il s'agit, ce qui n'est pas possible sur la base des données recueillies.

En reprenant la structure du deuxième facteur de l'analyse, on se rend compte en effet que ces deux facteurs (le 2 et le 4) sont fortement complémentaires: le second facteur opposait les communes encore fortement agricoles aux autres, le quatrième distingue parmi ces «autres» celles qui sont tertiaires de celles qui sont secondaires. Parallèlement, cela signifie qu'il y a une indépendance entre l'habitat et le lieu de travail et que les typologies des communes ne sont pas liees: connaître la structure de la population d'une commune ne permet pas d'anticiper la structure de ses emplois. D'une certaine manière, il existe deux Suisses urbaines: une Suisse des habitants, une Suisse des travailleurs, dont les différenciations sont indépendantes l'une de l'autre.

L'analyse de la répartition spatiale des communes (carte 4) selon leur comportement sur le quatrième facteur est moins évidente que celles des facteurs précédents. Il en ressort que la Suisse urbaine connaît une double différenciation entre secondaire et tertiaire, une différenciation entre agglomérations, une différenciation entre communes d'une même agglomération. On notera d'abord que l'opposition tertiaire/secondaire permet de retrouver ensemble, sur le pôle positif, les cinq grandes villes, Bâle devant à sa chimie d'être un peu plus équilibrée entre les deux secteurs $(0,79)$. Certaines de leurs banlieues sont nettement plus tertiaires, mais surtout par absence de secondaires et d'ouvriers. Quant aux communes de tête dans la hiérarchie des scores, elles relèvent toutes d'un tertiaire touristique.

Au niveau régional, tant les grandes régions d'industrie que les centres essentiellement tertiaires se distinguent plus ou moins bien. Au niveau régional, on constate une forte présence de l'industrie dans l'aire métropolitaine zurichoise, notamment dans sa partie ouest et sud (Argovie, Schwyz, Wetzikon-Pfäffikon, plus loin Lucerne et Stans), dans l'est de la Suisse en général, dans l'arc jurassien et la région bâloise. Au contraire, les activités tertiaires sont nettement dominantes dans les régions de Berne (administration fédérale oblige), dans l'arc lémanique et au Tessin. Au niveau macro-régional, on retrouve une opposition entre cœur industriel de la Suisse, autour de Zurich notamment, et une Suisse occidentale (Suisse romande et Berne) et au Tessin. Les Alpes connaissent une situation hybride entre fonds de vallée fortement industrialisés (Monthey, Viège, Coire par 
exemple) et les villes à vocation plus touristique (Davos, Interlaken).

$\mathrm{Au}$ niveau local, les partitions sont nettement plus franches. Au sein des grandes agglomérations, les communes centrales sont systématiquement plus tertiaires que le reste de l'agglomération. Il faut descendre assez bas pour trouver des communes centrales qui sont autant ou plus industrielles que leurs banlieues: la première est Baden, encore que celle-ci soit nettement moins industrielle que certaines de ses voisines. De manière générale, plus on descend dans la hiérarchie urbaine, moins les distinctions centre-périphérie sont fortes: on passe donc insensiblement des grandes agglomérations différenciées à de plus petites agglomérations qui se distinguent les unes des autres. Parmi ces petites agglomérations, certaines sont fortement industrielles; c'est le cas de la majorité des petites agglomérations de Suisse orientale (Wil, Uzwil, Arbon-Rorschach, Heerbrugg-Altstätten, Romanshorn-Amriswil), ainsi que de celles de l'arc jurassien (La Chaux-de-Fonds-Le Locle, Granges (SO), Neuchâtel, Soleure...). En revanche, à l'exception de certains cas particuliers (tourisme ou forte présence de l'armée), aucune petite agglomération n'est essentiellement tertiaire. On retrouve ici le résultat essentiel d'une autre étude, dynamique celle-là, du système urbain helvétique, significativement intitulée: «tertiairisation sélective et industrialisation diffuse» (CUNHA et RACINE 1992). Au total, une triple opposition régionale et locale, entre agglomérations plus ou moins industrielles d'une part, à l'intérieur des plus grandes, entre banlieues et centres d'autre part, entre types de banlieue enfin.

\section{Le genre comme facteur de différenciation: des régions historiquement féminisées?}

L'examen des variables (tableau 5) concernées par le cinquième facteur ne laisse aucun doute quant à l'opposition qu'il exprime: celle-ci concerne essentiellement la présence des femmes dans la population active. Le cinquième facteur oppose essentiellement les lieux où la population active est fortement féminisée dans le tertiaire et le primaire, des lieux où elle est restée avant tout masculine. Premier sujet d'étonnement, la proportion de femmes dans la population active, pourtant préférentiellement tertiaire à l'échelle nationale, ne s'inscrit que faiblement dans la structure des facteurs précédents. Elle n'est ni liée à la situation sociale, ni au genre d'emploi: de fait elle forme une dimension indépendante.

Second sujet d'étonnement, là où les femmes sont fortement présentes, elles le sont dans tous les secteurs de l'économie, comme le montre la présence du même côté du facteur de la proportion des femmes dans le tertiaire et dans le primaire. Cette proportion est également forte, que l'on se trouve au lieu de travail ou au lieu de domicile, ce qui signifie soit que les femmes "pendulent" peu, soit que cette opposition se traduit géographiquement par une opposition entre régions plus qu'entre communes. Troisième sujet d'étonnement, s'il semble que d'après le quatrième facteur, les femmes soient mieux représentées dans le tertiaire que dans les autres domaines économiques, elles le sont avant tout dans les lieux qui ne sont pas très tertiaires, comme le montre l'opposition dans ce facteur entre la part des femmes actives dans ce secteur et l'importance de ce dernier dans l'économie du lieu. En clair, ceci signifierait que les femmes sont prépondérantes dans le tertiaire là où il y en a peu, mais que là où le tertiaire domine, il est d'abord aux mains des hommes: comme si on ne laissait le tertiaire aux femmes que lorsque ce dernier ne correspond qu'à une activité annexe. Ce qui revient probablement à dire que les emplois tertiaires occupés par les femmes le sont dans le tertiaire banal bien plus que dans le tertiaire supérieur.

La répartition spatiale des communes selon leur «degré de féminisation» de la population active tel qu'exprimé par le cinquième facteur de l'analyse permet de reconnaître clairement plusieurs régions dont le comportement diffère des autres. La quasi-totalité des régions à forte féminisation de la population active se trouvent dans la moitié nord du pays. On y trouve essentiellement les foyers historiques de certaines industries en Suisse, celles justement qui tendent à occuper une main-d'œuvre féminine: ainsi, les agglomérations de Suisse orientale dans leur quasi-totalité, historiquement dévolues à l'industrie textile, comptent une forte proportion de femmes dans la population active. C'est également le cas des centres horlogers, comme La Chaux-de-Fonds, Le Locle ou Granges (SO), et de manière générale l'arc jurassien. A noter que des centres d'industrie légère, notamment dans l'arrière-pays bâlois et dans le canton d'Argovie, sont également bien féminisés. Ce qui conforte l'hypothèse selon laquelle la proportion de femmes dans la population active dépend largement de facteurs historico-culturels, puisqu'elle est particulièrement forte dans les régions où le travail féminin a une existence ancienne, dû à la présence historique d'industries à main-d'œuvre féminine, même si ces industries ont aujourd'hui partiellement disparu.

On pourrait reprendre ce facteur historique pour expliquer la faible féminisation d'autres régions du pays. Parmi les régions dont la main-d'œuvre est faiblement féminine, les zones industrielles classiques des grandes agglomérations. C'est le cas de la couronne nord de Zurich, des ouest lausannois et genevois, du nord-est bernois, et à un degré moindre, des industries bâloises, ou encore de l'agglomération de Coire: le domaine de l'industrie lourde reste masculin. On notera également la position particulière du Tessin, peu féminisé: une caractéristique qu'il est tentant de rattacher à la culture du pays.

Mais contrairement à ce que nous pouvions attendre de résultats obtenus ailleurs, ce facteur féminin n'indexe pas (encore?) un quelconque phénomène métropolitain: les grandes villes sont jusqu'ici relativement peu concernées (maximum à Bâle 0,54 , minimum à Zurich à - 0,20 ), une centaine de communes seulement se situent à plus ou moins un écart-type et parmi celles-ci rien n'indique une spécificité métropolitaine. A quelques exceptions près, telle la spécialisation horlogère au Locle, il s'agit de petites communes, les localisations pouvant relever, à cette 


\section{Géographie de la force de travail de la Suisse urbaine, 1990}

(805 unités spatiales et 46 caractéristiques)

Analyse en composantes principales, solution Varimax à 5 composantes, $62.9 \%$

\section{Composante 2}

Variance expliquée : $13.6 \%$

Variance cumulée: $\quad 35.7 \%$

\section{Structure}

Caractéristiques

Employés au trav.

Communes d'emploi

Densité

Etrangers

Actifs féminins au dom.

Prof. intell. et d'enc. au trav.

Prof. intermédiaires au trav.

Employés au dom.

Non qualifiés au dom.

Taux d'activité

Travailleurs tertiaires au trav.
Saturations +

Profil corrélatif des variables sur l'axe
0.73
0.63
0.62
0.62
0.56
0.55
0.52
0.41
0.40
0.39
0.37

Rapport de dépendance

Travailleurs primaires au dom.

Indépendants au dom.

$-0.38$

Travailleurs primaires au trav $\quad-0.78$

Indépendants au travail $\quad-0.84$

Saturations -

\section{Poids locaux}

Projection des unités spatiales sur l’axe

Unités spatiales

1. Genève

2. Meyrin (Genève)

3. Vevey

4. Basel

5. Renens (Lausanne)

7. Kloten (Zürich)

10. Paradiso (Lugano)

11. Zürich

13. Lausanne

25. Bern

43. Birsfelden (Basel)

55. Ostermundigen (Bern)
Ecarts-types

2.94

2.21

2.18

2.17

2.13

2.06

2.04

2.03

1.97

1.79

1.55

1.44
540. Einsiedeln

743. Hochwald (Basel)

761. Unterlunkhofen (Zürich)

771. Grafenried (Bern)

788. Poliez-le-Grand (Lausanne)

800. Arnex / Nyon (Genève)

801. Courtion (Fribourg)

802. Arconciel (Fribourg)

803. Gerlikon (Frauenfeld)

804. Niederwil (Frauenfeld)

805. Villarsel / Marly (Fribourg)
$-0.43$

$-1.47$

$-1.60$

$-1.70$

$-2.04$

$-2.51$

$-2.52$

$-2.60$

$-2.68$

$-2.73$

$-5.07$

\section{Définition de la composante}

Les centres d'emplois urbains et leurs corrélatifs (densité, étrangers) versus périphéries «rurbaines»

\section{IDENTIFICATION}

Centralité différentielle des communes 


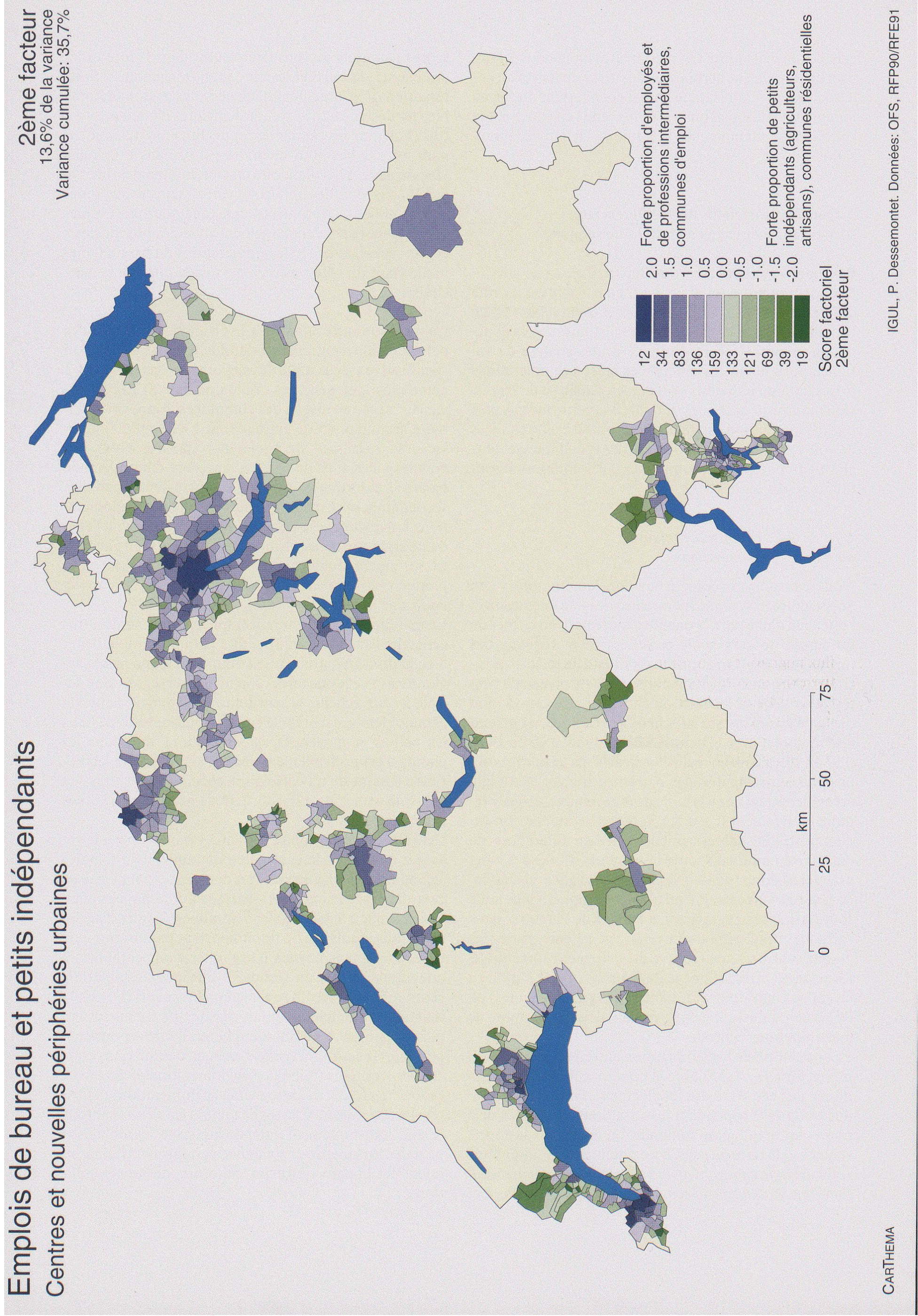


échelle, de l'aléatoire. On reconnaîtra en revanche l'impact historique que certaines industries peuvent avoir sur la société, en féminisant le travail et en faisant du travail féminin un fait social qui n'est plus remis en cause par la suite, indépendamment de la survie du facteur de féminisation initial.

\section{Quelques corrélatifs de la différenciation socio-spatiale des agglomérations helvétiques}

Revenant à nos hypothèses de départ, il n'est pas sans intérêt de reprendre ici, thème à thème, l'analyse du rôle différenciateur des tailles urbaines et éventuellement de l'appartenance régionale et/ou linguistique dans la dynamique urbaine globale. Couplée à une analyse de variance portant sur les différences entre agglomérations prises comme individus statistiques, l'analyse de régression montre qu'en termes de taille, il se confirme que plus une agglomération est importante, plus il y a de chances de voir y apparaître des phénomènes spécifiques, et plus elle est grande, plus les communes qui la composent sont différenciées entre elles.

\section{Effet de taille et centralité relative au sein des agglomérations}

Nous avons déjà évoqué le lien banalement observé en Suisse entre le système hiérarchisé des lieux centraux et l'intensité d'une part, la qualité d'autre part, en termes de formation, de statut socio-professionnel et d'origine, des flux migratoires. On connaît par ailleurs la relation positive existant entre le gradient mesurant le niveau de centralité urbaine et les taux de chômage, une corrélation mesurée pour l'instant à l'échelle des cantons et qui tend à se renforcer avec le temps: 0,27 en 1988, 0,42 en 1991, 0,57 en 1992, 0,60 en mai 1994. Précisons ici que la composante centralité dégagée par notre analyse factorielle est évidemment liée à la taille de la commune, sans s'y réduire entièrement $(r=0,71)$, la part de variance non-expliquée traduisant essentiellement les différences de maillage communaux entre les cantons. Treize des vingt premières communes obtenant des résidus élevés indexant une surcentralité relative par rapport à leur taille sont tessinoises et gravitent, à l'image de Paradiso, dans l'aire métropolitaine de Lugano. Par ailleurs, parmi les grands centres, seule Genève est nettement surcentrale, les autres centres, à l'image de Lausanne et de Zurich en faveur de Chavannes et Ecublens (les grandes écoles) ou de Kloten, subissant déjà l'exurbanisation de nombre de leurs fonctions centrales.

La population de l'agglomération entretient une relation moins forte $(r=0,55)$ avec la composante socio-économique des cadres et des intellectuels, d'importants résidus traduisant cependant les variations régionales déjà notées entre la Suisse romande, où elles sont surreprésentées, et le reste du pays. Il n'en demeure pas moins que l'effet de taille en regard de la première composante sociale indexant le statut socio-économique est indiscutable. Les scores factoriels moyens décroissent dans l'ordre exact des classes de taille et les cinq grandes agglomérations ressortent très largement détachées des agglomérations plus modestes, cette moyenne des scores n'étant positive $(0,72)$ qu'avec les premières, et déclinant systématiquement vers le bas pour atteindre $-0,70$ dans le cas des villes isolées. A l'évidence les classes dominantes du pays ne sont pas réparties uniformément au sein des agglomérations suisses, concentrées dans quelques-unes seulement (Genève arrivant largement en tête, à près de deux écarts-types), toutes les agglomérations du bassin lémanique étant du côté positif, ainsi que la métropole zurichoise.

L'opposition entre Suisse des arts et métiers et Suisse des petites mains n'entretient pratiquement pas de relation significative avec la taille des agglomérations $(r=0,12)$, à peine plus avec celle des communes $(r=0,30)$. En revanche, saisie au niveau des communes, l'opposition tertiaire/secondaire reste marquée entre les emplois de services se localisant de préférence dans le cœur des grandes villes ainsi que, subsidiairement dans la grande banlieue, alors que les couronnes sont le fief des activités secondaires, et ce quelle que soit la taille de l'agglomération. La relation est donc complexe, traduisant l'effet d'une structure concentrique: fortement tertiaire au centre, fortement secondaire dans le péricentre que forme la première couronne historique, plutôt tertiaire dans la couronne suivante.

En matière d'emploi féminin enfin, si, d'une manière très générale, l'analyse confirme que la taille stimule bel et bien la discrimination entre communes d'une même agglomération et donc un certain niveau de ségrégation sociale à cette échelle, la sensibilité relativement faible de l'emploi féminin à la taille (un peu plus développé dans les petites et moyennes agglomérations que dans les grandes) est probablement liée au fait de l'assise territoriale classique du travail féminin en Suisse, l'arc jurassien et la Suisse orientale qui abritent de nombreuses petites agglomérations.

Cet effet de taille n'épuise pas l'analyse que l'on peut prolonger en termes de centralité différentielle au sein des agglomérations. En classant les communes par niveau de centralité (communes périphériques, communes appartenant à une zone centrale, communes noyaux d'agglomérations bipolaires ou ne comportant pas de zone centrale, communes noyaux d'agglomérations comportant une zone centrale), on découvre l'effet incontestable de la centralité, mais sur un seul facteur, le second ( $F=94,4)$. Relation tautologique en fait puisque ce facteur discrimine justement les communes centrales des communes périphériques. En revanche, l'examen des relations entre ces deux critères (taille de l'agglomération et niveau de centralité au sein de cette même agglomération) peut se révéler intéressant. Classes de taille et classes de centralité sont statistiquement indépendants; leur combinaison peut dès lors mettre en évidence certains faits qui ne seraient pas apparus jusqu'ici, en termes de sous-populations. 
On constate ainsi que les classes dominantes indexées par le premier facteur se concentrent essentiellement dans les périphéries des grandes agglomérations, et de manière résiduelle dans les zones centrales de ces dernières; elles sont par contre absentes des centres des petites et moyennes agglomérations. Les constatations faites sur le second facteur (centre-périphérie) sont assez banales, comme on pouvait s'y attendre; les classes moyennes associées au troisième facteur se concentrent également dans les banlieues et évitent nettement les centre-villes des agglomérations. Les dichotomies du quatrième facteur (industrie-services) confirment le fait que les services se localisent de préférence dans le centre des grandes villes ainsi que, subsidiairement, dans la grande banlieue, alors que les couronnes sont le fief des activités secondaires, indépendamment de la taille de l'agglomération. On constate enfin que l'emploi féminin du cinquième facteur est systématiquement plus fort dans les centres que dans les banlieues, et systématiquement plus fort dans les petites agglomérations que dans les grandes.

La taille de l'agglomération induit-elle une plus forte discrimination entre communes? L'observation de la répartition des communes d'une agglomération sur chaque facteur par le moyen de l'écart-type devrait permettre de répondre: plus celui-ci est grand, plus la dispersion des communes de l'agglomération (et donc les dichotomies internes à cette dernière) seront fortes. Sur le premier facteur, on constate que les communes des cinq grandes montrent une plus forte dispersion que celles qui appartiennent $\grave{a}$ d'autres sous-groupes (écart-type de 1,03 contre, en gros, 0,6 pour les autres catégories), ce qui semble indiquer que la discrimination sociale que représente ce premier facteur $y$ est plus forte. L'examen agglomération par agglomération confirme ce résultat liminaire, puisque toutes les grandes agglomérations montrent une forte dispersion de leurs communes sur ce facteur. C'est dire que du point de vue social, l'hypothèse est confirmée.

Le résultat est moins net sur le second facteur indexant la centralité interne des agglomérations. Toutes les catégories montrent grosso modo la même dispersion, ce qui semble indiquer que la structuration entre centre et périphérie se manifeste partout de manière à peu près similaire, l'examen agglomération par agglomération confirme ce point de vue et montre également une très forte dispersion pour pratiquement chaque agglomération du pays. Autant dire qu'à quelques rares exceptions près, en termes d'activités, les agglomérations de ce pays seraient fortement différenciées. L'hypothèse selon laquelle plus une agglomération est grande, plus la différenciation interne l'est également, est ici infirmée, sauf à se souvenir que la centralité était un peu plus forte dans les grandes agglomérations que dans les petites et moyennes.

Sur le troisième facteur, il semble, à l'instar du premier, que la discrimination entre classes moyennes et classes servantes de la population soit un peu plus forte dans les grandes et moyennes agglomérations que dans les petites, mais on constate à l'examen des dispersions agglomération par agglomération que les situations sont très variables. De manière générale, les dispersions sont assez faibles (plus fortes cependant en Suisse latine), ce qui signifie que c'est bien entre agglomérations, et surtout entre régions que la discrimination principale intervient, et que la Suisse alémanique est décidément plus homogène que la Suisse latine.

Comme déjà souligné, le quatrième facteur (industrieservices) ne montre pas du tout d'effet de taille; la dichotomie n'est pas plus forte dans les grandes agglomérations. Le cas du cinquième facteur est assez similaire au troisième, en encore plus flou: ici aussi, la discrimination régionale est prépondérante.

\section{L'effet régional}

L'analyse de variance montre que si les variations régionales ne jouent pratiquement aucun rôle sur le facteur de centralité ( $F$ non significatif), ce qui semble signifier que si les agglomérations helvétiques se structurent sur le même modèle d'un bout à l'autre du pays, il n'en va pas de même en ce qui concerne les dimensions découvertes en termes de classes socio-spatiales.

L'opposition entre tertiaire et secondaire n'implique guère de variations régionales, sinon au niveau de toutes petites agglomérations, tant du côté industriel (Granges, Arbon-Rorschach, Uzwil) que du côté tertiaire (les spécialisations touristiques comme à Davos ou Interlaken). En revanche l'opposition entre Suisse des arts et métiers et Suisse des petites mains se traduit par des variations plus fortes, mettant d'abord en évidence une claire opposition entre le cœur économique du pays (le Triangle d'Or) et les périphéries, latines notamment (Suisse romande et Tessin). Quant à l'emploi féminin, tel qu'indexé par la cinquième composante, il varie assez nettement d'une agglomération à l'autre, du fait en particulier du fort emploi féminin dans le bassin horloger, ainsi qu'en Suisse orientale (textile) alors qu'il est très faible au Tessin: un facteur régional évidemment lié aux spécialisations industrielles traditionnelles des bassins concernés.

Regroupés sur une base linguistique, les résultats confirment par ailleurs que les variations régionales touchent avant tout la composante indexant l'opposition entre classes dominantes et ouvriers, la Suisse romande, aux proportions toujours plus grandes de cadres et d'intellectuels, se détachant largement du reste du pays, en opposition avec la Suisse alémanique et le Tessin. Mais la différenciation la plus nette concerne la deuxième composante sociale (3ème facteur), la découverte en termes non seulement socio-économiques mais proprement géographiques d'une Suisse dite des "arts et métiers» marquant la supériorité en la matière de la Suisse alémanique, nettement mieux dotée en classes moyennes que le reste du pays, de part et d'autre d'une frontière bien marquée avec à la Suisse latine. Une nouvelle expression du fameux «rideau de rösti» (RACINE 1994) si fréquemment évoqué au 


\section{Géographie de la force de travail de la Suisse urbaine, 1990}

( 805 unités spatiales et 46 caractéristiques)

Analyse en composantes principales, solution Varimax à 5 composantes, $62.9 \%$

\section{Composante 3}

Variance expliquée : $10.9 \%$

Variance cumulée : $\quad 46.6 \%$

\section{Structure}

Caractéristiques

Form. professionnelle

Prof. intermédiaires au dom.

Revenus entre 50 et $75 \mathrm{kF}$

Prof. intermédiaires au trav.

Taux d'activité

Actifs féminins primaires au trav

Acrifs féminins primaires au dom.0.44

Form. professionnelle sup.

Employés au dom.
Saturations +

Profil corrélatif des variables sur l'axe

0.80

0.71

0.57

0.48

0.48

0.44

0.42

0.41
Revenus entre 20 et $30 \mathrm{kF}$

Revenus inférieurs à $20 \mathrm{kF}$

Etrangers

Non qualifiés au dom.

Non qualifiés au travail

Form. scolaire obligatoire
$-0.36$

$-0.37$

$-0.52$

$-0.53$

$-0.63$

$-0.70$

Saturations -

\section{Poids locaux}

Projection des unités spatiales sur l’axe

Unités spatiales

1. Regensberg (Zürich)

2. Greifensee (Zürich)

3. Widen (Zürich)

4. Bonstetten (Zürich)

5. Schwerzenbach (Zürich)

8. Henggart (Winterthur)

14. Urtenen (Bern)

18. Giebenach (Basel)

78. Froideville (Lausanne)

177. Puplinge (Genève)

196. Baden

310. Bern

402. Zürich
Ecarts-types

2.49

2.34

2.13

2.07

2.06

2.00

1.86

1.77

1.29

0.88

0.78

0.38

0.05
444. Küsnacht (Zürich)

524. Wünnewil Flamatt (Bern)

571. Basel

683. Lausanne

744. Genève

785. Crissier (Lausanne)

787. Chiasso

801. Tenero-Contra (Locarno)

802. Vandoeuvres (Genève)

803. Muralto (Locarno)

804. Cologny (Genève)

805. Roveredo (Lugano)
$-0.10$

$-0.45$

$-0.63$

$-1.08$

$-1.50$

$-1.96$

$-2.02$

$-2.41$

$-2.48$

$-2.50$

$-2.51$

$-2.68$

\section{Définition de la composante}

La Suisse des «Arts et Métiers» ou «classe moyenne» versus la Suisse des «petites mains» ou «classe servante»

\section{IDENTIFICATION}




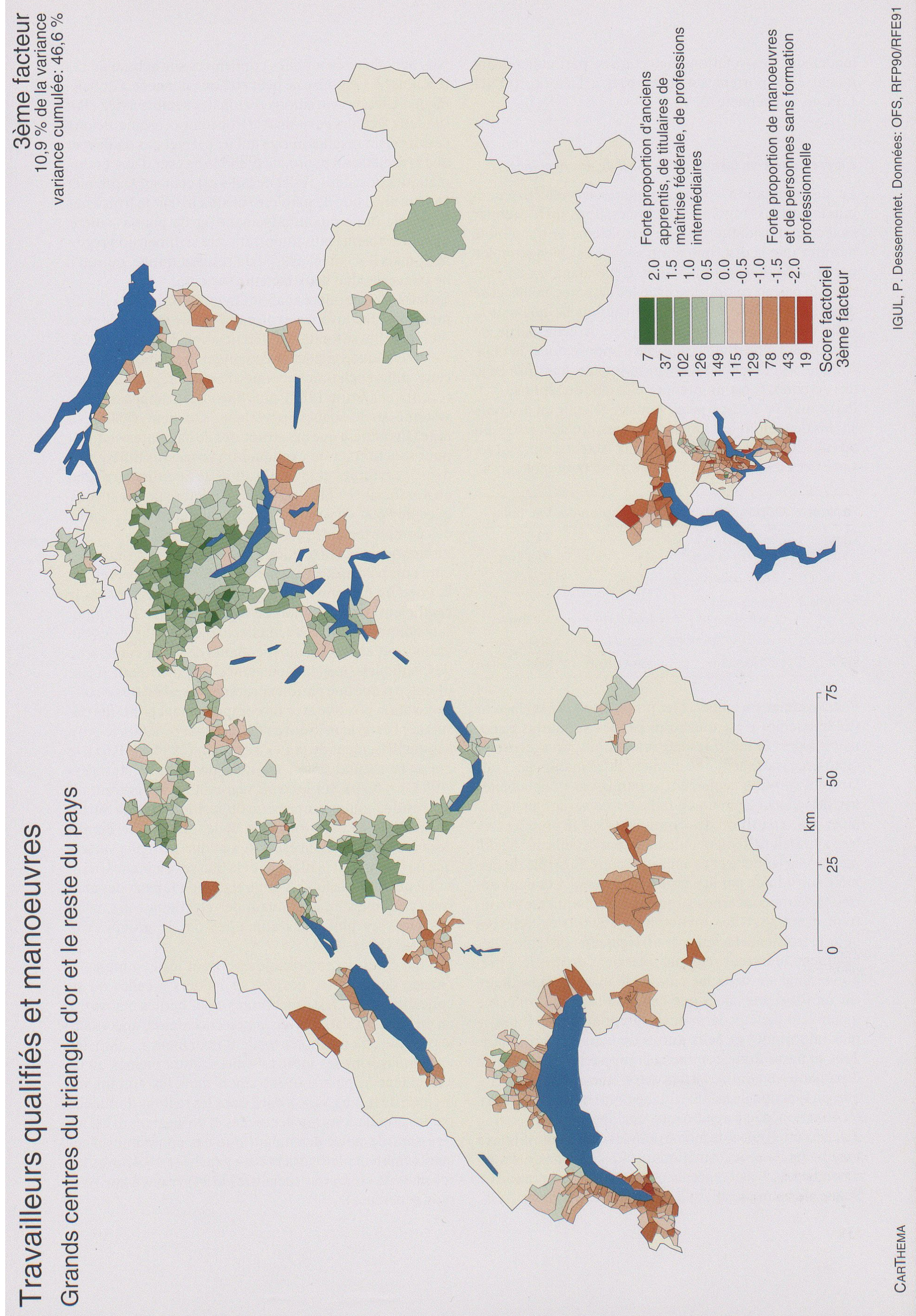


lendemain des votations helvétiques, particulièrement quand elles portent sur le rapport à l'altérité (ONU, Europe, citoyenneté)?

\section{En première conclusion: qu'en est-il de nos hypothèses?}

La discrimination entre communes est effectivement plus forte dans les grandes agglomérations sur le premier facteur, et subsidiairement sur le troisième, ce qui semble montrer que la discrimination sociale est bien stimulée par la taille de l'agglomération. Cependant, un tel effet n'existe pas en ce qui concerne les activités: celles-ci se discriminent avec la même intensité quelle que soit la taille de l'agglomération concernée. A cette partie de l'hypothèse, une double réponse s'impose donc: la taille stimule bel et bien la ségrégation sociale, mais pas celle des activités. C'est un premier élément de réponse.

Parvenus à ce stade, il serait tentant de tenir un discours sur les deux premières hypothèses, en croisant les résultats des études menées sur ces deux facteurs. On peut résumer ces résultats sous la forme d'un petit tableau:

\begin{tabular}{llll}
\hline Facteur & Opposition & Var. régionale & Var. taille \\
\hline Facteur 1 & $\begin{array}{l}\text { classe aisée/ } \\
\text { reste de la pop. }\end{array}$ & assez forte & très forte \\
Facteur 2 & centre/périphérie & nulle & nulle \\
Facteur 3 & $\begin{array}{l}\text { arts-métiers } \\
\text { cl. servantes }\end{array}$ & très forte & rel. forte \\
Facteur 4 & industrie/services & assez faible & nulle \\
Facteur 5 & fact. féminin & assez forte & assez faible \\
\hline
\end{tabular}

Pour résumer, on constate les points suivants: un facteur (le deuxième) n'est touché ni par les variations régionales, ni par la taille des agglomérations, un second (le quatrième) ne l'est que de manière assez marginale. Trois facteurs sur cinq seulement apparaissent donc affectés par des effets généraux de taille ou de variations régionales. La répartition des communes sur le premier facteur apparait nettement influencée par la taille de l'agglomération à laquelle elles appartiennent, ainsi que de manière subsidiaire par le paramètre régional. Les deux autres facteurs concernés (troisième et cinquième) montrent avant tout des dichotomies régionales, relayées pour le troisième facteur par un léger effet de taille. Globalement, le facteur régional intervient de manière prépondérante sur deux facteurs et de manière subsidiaire sur un troisième, alors que l'effet de taille ne concerne qu'un seul facteur de manière prépondérante (mais le plus important), et deux autres de manière subsidiaire. Tout se passe donc comme si la proportion des classes dominantes en Suisse variait selon une double thématique: en fonction de la taille de l'agglomération d'une part, en fonction de la région qui englobe l'agglomération d'autre part. Il en va de même pour les classes moyennes à forte proportion de formations professionnelles, mais encore une fois, l'effet régional et leur surreprésentation en Suisse alémanique affaiblit la corrélation simple.
Nos hypothèses sont-elles confirmées sur la base de ces résultats? La réponse ne peut être que nuancée. Globalement, l'effet des variations régionales semble assez clair sur deux facteurs au moins (le troisième et le cinquième), ce qui est un résultat mitigé qui ne permet pas d'affirmer que la dimension régionale prend le pas sur d'autres thématiques possibles; l'effet de taille est clair sur le premier facteur, ainsi que de manière subsidiaire sur le troisième. Il semble donc quantitativement (et de par sa netteté, qualitativement) plus important que la dimension régionale, mais il s'agit ici aussi d'un résultat mitigé, puisqu'il ne concerne que deux facteurs sur cinq.

En faisant en dernière analyse l'hypothèse que les cinq dimensions découvertes expriment les structures de base sur lesquelles se fonde la différenciation de la force de travail helvétique, on peut se demander dans quelle mesure cette différenciation recoupe d'autres représentations (CUNHA et RACINE 1987), plus fines, des composantes du système socio-spatial helvétique. On peut également faire l'hypothèse que ces structures de base interviennent pour sanctionner ou expliquer un processus différentiel dans les rythmes et la localisation de la croissance, tout en s'associant enfin à certaines caractéristiques spécifiques de l'état civil et de l'origine ethnique, voire, enfin, des comportements politiques.

La matrice des corrélations associant deux à deux nos cinq composantes à cet ensemble de variables révèle en fait peu de liaisons vraiment significatives dépassant le coefficient de corrélation de 0,5 . Il reste que la composante opposant la Suisse intellectuelle à la Suisse des ouvriers, tout en restant indépendante de la majeure partie des variables (une indépendance qui mériterait d'être discutée parce que parfois surprenante) est en très étroite corrélation positive avec un comportement politique ouvert sur l'extérieur, le oui à l'EEE (1992: r =0,60), le oui à l'assouplissement de la Lex Friedrich (1994: $r=0,65)$, le oui à la naturalisation facilitée des jeunes étrangers (1994: $r=0,70)$. Sur les trois régressions, la distribution des résidus souligne une nette opposition entre la Suisse occidentale (Suisse romande, Bâle, Soleure et Berne) dont le comportement est systématiquement plus ouvert que ne le voudrait le modèle, et la Suisse orientale. Un résultat qui tout à la fois recoupe et complète ceux des analyses conduites au lendemain des élections voire, ceux des analyses plus fines conduites par le groupe Vox (RACINE 1994).

De son côté, la composante opposant les communes à l'image de la Suisse des arts et métiers à celles où se concentrent les classes servantes et les petites mains, est en étroite corrélation avec la présence des étrangers sur le pôle négatif: nos hôtes tendent nettement à s'associer aux professions les moins formées, alors mêmes qu'ils s'installent le plus souvent sinon dans les communes mêmes, du moins, juste à côté, dans les régions dominées par les populations intellectuelles et dirigeantes. Il est intéressant de noter cependant que ces communes d'accueil semblent plutôt orientées à gauche $(r=0,42$ avec les résultats de l'initiative socialiste (1994) visant à une AVS plus généreuse). 


\section{Typologie des communes d'agglomération selon leur force de travail}

La géographie suisse dispose déjà d'un excellent essai sur la diversité des communes de ce pays et les types qui peuvent en résulter en partant des points de vue de l'économie, de l'habitat, des tendances démographiques, de l'importance enfin des mouvements pendulaires (JOYE et SCHULER 1992). Il concerne l'ensemble des 3021 communes et repose sur une série d'indicateurs qui ont l'avantage de prendre en compte la fonction des communes telle qu'elle est déterminée par ses liens avec celles qui l'entourent. Centres, communes suburbaines, communes périurbaines, communes rurales à pendularité importante, communes industrielles et tertiaires, communes agro-industrielles et agro-tertiaires, communes agraires et communes touristiques se partagent l'espace helvétique. Image plausible et somme toute conforme à l'expérience de chacun, alors même qu'elle dérive d'une analyse multivariée sophistiquée.

Limitée au seul système urbain (les 805 communes dites d'agglomération), notre visée était autre, plus sensible en particulier aux contenus sociaux et économiques mais sans référence directe aux mouvements pendulaires autre qu'à travers la distinction entre saisie des données au lieu de résidence et saisie sur le lieu de l'emploi. Les cinq facteurs découverts traduisent autant de dimensions latentes, mathématiquement définies comme indépendantes, mais complémentaires et additives en fait de l'espace socio-économique des agglomérations helvétiques. Ainsi, les facteurs 1 et 3 sont clairement des facteurs de différenciation sociale, discriminant les actifs au lieu de domicile, en tant qu'habitants, selon leur position dans le marché de l'emploi et les communes selon leur profil sociologique. De même, les facteurs 2 et 4 sont des facteurs de différenciation économique, qui discriminent les actifs sur leur lieu de travail selon leurs branches d'activités, et les communes selon leur spécialisation économique. Quant au cinquième facteur, il met en évidence un phénomène indépendant lié à la différenciation de genre.

Il est tentant, dès lors, de créer deux typologies distinctes sur la base de ces facteurs: une typologie sociale prenant en compte les facteurs 1 et 3 , et une typologie économique se fondant sur les facteurs 2 et 4 . La méthode de classification utilisée est la Classification ascendante hiérarchique, appliquée sur la racine carrée de leur valeur absolue afin d'éviter que les outliers ne perturbent l'analyse; ces valeurs ont été ensuite multipliées par la valeur propre de leurs facteurs respectifs afin de maintenir le principe de hiérarchie entre les dimensions découvertes.

\section{Première classification: la typologie sociologique}

Combinant l'opposition entre communes à forte proportion de classes dominantes et communes à forte proportion d'autres classes d'une part, et l'opposition entre classes moyennes et "classes (dites) servantes" d'autre part, une solution à cinq types se regroupant en deux ensembles principaux a èté retenue (carte 5).

Le premier de ces deux ensembles est formé de deux types ayant en commun le fait de contenir les communes qui n'ont qu'une faible proportion de classes dominantes en leur sein. 472 des 805 communes de l'analyse font partie de l'un ou l'autre. 226 communes font partie du premier type, qui se manifeste par un score négatif sur le premier et le troisième facteur, à savoir une absence de classes dominantes, ainsi qu'une absence de classes moyennes; c'est dire que ce premier type est formé des communes qui abritent une très forte proportion de population appartenant à la classe servante. Il s'agit d'un ensemble de communes abritant une population à "statut socio-résidentiel modeste». Le second type englobe 246 communes, et se distingue du premier par son score positif sur le troisième facteur: c'est dire que dans ces communes, tant la proportion de classes dominantes que de classes servantes est faible et que ce sont les classes moyennes qui y dominent très nettement: nous avons donc baptisé ce nouveau type comme celui des «communes à statut socio-résidentiel homogène».

Le second groupe est formé des trois types restants; il se distingue du premier groupe par le fait que dans chacun de ces trois types, la proportion des dominants est nettement supérieure à la moyenne. 333 communes d'agglomération font partie de ce groupe. La majorité d'entre elles font partie d'un type contenant 178 communes, se distinguant par ses scores positifs sur les deux facteurs, à savoir qu'aussi bien la proportion de classes dominantes que celle des classes moyennes y sont supérieures à la moyenne. Des communes qui se distinguent donc, a contrario, par la présence comparativement faible des populations servantes: nous évoquerons à cet égard des "communes à statut socio-résidentiel aisé». Les deux derniers types sont assez proches l'un de l'autre, et ont en commun de contenir les communes qui se profilent positivement sur le premier facteur et négativement sur le troisième, ce qui signifie une forte présence des classes dominantes comme des classes servantes, avec une faible présence des classes moyennes, ce qui est bien le fait de sociétés locales duales. Cet ensemble est d'ailleurs partagé en deux types: le premier, contenant 75 communes, a été baptisé "communes à statut socio-résidentiel dual»; le second de ces deux types, concernant 80 communes, se distingue du premier par le fait que les classes dominantes y sont nettement mieux représentées; il regroupe un ensemble de communes baptisé "communes à statut dual aisé».

\section{Une suisse alémanique homogène ou aisée versus une Suisse latine duale et modeste?}

L'examen de la répartition spatiale de ces cinq types permet de regrouper d'autres ensembles et sous-ensembles à travers deux séries de distinctions complémentaires: une analyse type par type, puis une analyse géographique des grandes répartitions territoriales. 


\section{Géographie de la force de travail de la Suisse urbaine, 1990}

( 805 unités spatiales et 46 caractéristiques)

Analyse en composantes principales, solution Varimax à 5 composantes, $62.9 \%$

\section{Composante 4}

Variance expliquée: $\quad 10.6 \%$

Variance cumulée : $\quad 57.2 \%$

\section{Structure}

Caractéristiques

Travailleurs tertiaires au trav.

Actifs féminins au trav.

Services sociaux

Travailleurs tertiaires au dom.

Actifs féminins tertiaires au trav

Employés au travail
Profil corrélatif des variables sur l'axe

Saturations +
Non qualifiés au trav.

Taux d'activité

Travailleurs secondaires au dom.

Industrie forte

Ouvriers au travail

Travailleurs secondaires au trav.
$-0.37$

$-0.39$

$-0.50$

$-0.53$

$-0.60$

$-0.80$

Saturations -

\section{Poids locaux}

Projection des unités spatiales sur l'axe

Unités spatiales

1. Scherzingen (Kreuzlingen)

2. Orselina (Locarno)

3. Unterseen (Interlaken)

4. Lopagno (Lugano)

5. Brione / Minusio

8. Bettingen (Basel)

11. Echichens (Lausanne)

21. Bremgarten / Bern (Bern)

25. Genolier (Genève)

27. Lugano

42. Zollikon (Zürich)

49. Lausanne

53. Bern

85. Zürich

91. Genève

170. Basel
Ecarts-types

3.14

3.08

2.74

2.55

2.53

2.32

2.02

1.86

1.78

1.77

1.62

1.56

1.48

1.20

1.18

0.79

\section{Uzwil}

765. Bäriswil (Bern)

769. Kaiseraugst (Basel)

792. Bergdietikon (Zürich)

800 . Satigny (Genève)

801. Felben-Well. (Frauenfeld)

802. Carabbia (Lugano)

803. Birr (Brugg)

804. Senèdes (Fribourg)

805. Mex (Lausanne)
$-1.45$

$-1.59$

$-1.70$

$-2.25$

$-2.90$

$-2.95$

$-3.18$

$-3.48$

$-3.52$

$-3.80$

\section{Définition de la composante}

Communes d'emplois tertiaires versus communes d'emplois secondaires 


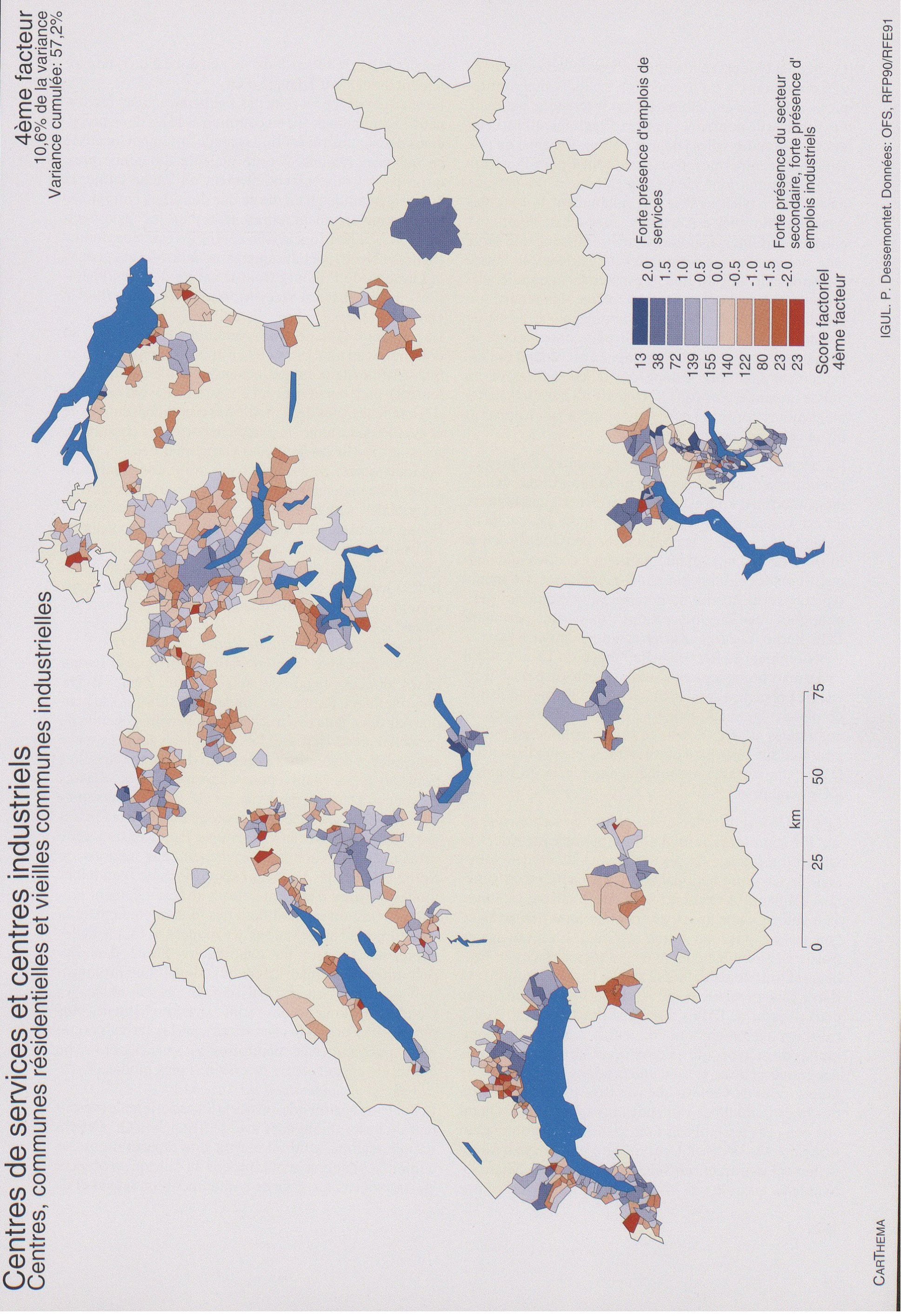


Le premier type est celui des structures modestes. Avec ses 226 communes, il est le seul dont les individus sont répartis à peu près équitablement sur le territoire national. On y trouve de nombreux centres d'agglomération: deux des cinq grandes villes (Bâle et Lausanne), ainsi qu'une kyrielle de centres moyens à travers tout le pays. Plusieurs agglomérations font même intégralement ou presque partie de ce type: c'est le cas notamment le long des Alpes, de Monthey à Altstätten, ainsi qu'au Tessin; par ailleurs, certaines agglomérations y laissent une large part, notamment les périphéries industrielles en Suisse romande et le long du Jura. C'est bien là un premier élément de différenciation. De plus, même si la répartition générale est globalement assez homogène, force est de constater que certaines régions du pays sont plus concernées que d'autres: en Suisse romande en général (à l'exception de Genève), le long des reliefs, au Tessin, en Suisse orientale, alors que le Triangle d'Or est très nettement moins concerné par le phénomène qui n'apparaît qu'en certaines périphéries.

Le second type est celui des structures homogènes: contrairement au précédent, il ne se répartit pas de manière uniforme: s'il n'est présent qu'en certaines régions, il y est souvent dominant. Ce type compte également de nombreux centres d'agglomération: ceux de Zurich et Berne, ainsi qu'une grande partie des centres alemaniques, tous les centres zurichois notamment. Ce même type concerne encore de nombreuses communes périphériques des agglomérations, dans un périmètre Bâle-Berne-Lucerne-Zurich essentiellement. Il est souvent dominant de Berne à Schaffhouse, de Thoune à Wil (SG), mais pratiquement absent du reste du pays. S'il est encore présent ça et là en Suisse orientale (quoique non dominant), la Suisse latine ne compte que très peu de communes affiliées à ce type (une vingtaine en tout et pour tout sur 246). En d'autres termes, et pour l'essentiel, le fait d'une certaine Suisse alémanique, celle du Triangle d'Or notamment.

Le troisième type est celui des communes à structure aisée, 178 communes au total. Comme pour le précédent, la répartition spatiale de ces communes différencie fortement le territoire national, selon les mêmes axes généraux d'ailleurs. A savoir qu'on le trouve surtout en Suisse alémanique. Cette fois peu de centres appartiennent à ce type (citons pourtant Aarau et Zoug). En revanche, ce troisième ensemble couvre de grandes surfaces en banlieue, autour de Bâle (tout le sud de l'agglomération), de Berne (une première ceinture), et surtout de Zurich (les deux rives du lac, l'Albis, une partie du nord-ouest. A elles trois, ces agglomérations recèlent la majorité des communes de ce type, qui se développe surtout aux abords des grands centres. C'est d'ailleurs dans les banlieues de Lausanne et de Genève que l'on trouve la quasi-totalité des rares communes romandes lui appartenant. Quant aux deux cas tessinois, ils font partie de l'agglomération luganaise. On l'a déjà dit, ce type de communes est essentiellement représenté en Suisse alémanique: la Suisse romande en compte une vingtaine tout au plus, la Suisse orientale une, le Tessin deux: il s'agit donc d'un type clairement associé au Triangle d'Or.

Le quatrième type est celui des communes duales, regroupant 75 communes. Il a en commun avec les deux précédents d'avoir une répartition spatiale fortement localisée en quelques régions, à cette majeure différence près, qu'on les retrouve essentiellement en Suisse latine: la Suisse alémanique n'en recèle que deux. Les communes duales sont largement représentées dans les agglomérations de Genève (dont la ville-centre, les banlieues industrielles et Nyon), de Lausanne (autour de Morges et dans le Lavaux), de Fribourg (tout le centre de l'agglomération), de Neuchâtel, à Vevey-Montreux et Lugano (tout le centre ici aussi).

Le cinquième type, celui des communes duales aisées $(80$ communes au total), reprend en gros cette répartition en Suisse latine (deux communes en Suisse alémanique seulement), où il concerne tout d'abord Genève (l'essentiel des banlieues, plus de la moitié des communes suisses de ce type) et Lausanne (la grande majorité des communes restantes, les belles banlieues en premier lieu).

Au total, quatre de ces cinq types ayant une répartition territoriale nettement dissemblable, c'est dire qu'il existe de grandes disparités entre les régions urbaines de Suisse, ce qui signifie que les différences entre agglomérations sont au moins aussi importantes que les différences entre communes au sein d'une même agglomération. Quelques régions urbaines apparaissent ainsi comme nettement individualisées dans leurs contenus variés.

- Villes et agglomérations de Zurich-Triangle d'Or:

L'espace formé par l'agglomération zurichoise et ses annexes directes (aggl. de Winterthour, Zoug, Wetzikon-Pfäffikon, Rapperswil, Baden, Schaffhouse) se marque par deux faits essentiels: l'homogénéité générale de la société et sa richesse. Les communes de cet espace sont soit des communes aisées, soit des communes dites homogènes abritant pour l'essentiel des classes moyennes. Dans les deux cas, les classes dites servantes de la société y sont peu représentées: c'est dire la force et la cohésion de la société zurichoise. Par extension, cette caractéristique s'applique également aux régions de Berne et, avec des nuances (apparitions de communes modestes, voire d'agglomérations structurellement modestes, mais qui ne modifient pas fondamentalement la structure que nous venons d'évoquer), à toutes celles comprises au sein du quadrilatère Berne-Bâle-Zurich-Interlaken, quadrilatère qui connaît une prolongation dans les Grisons. Au sein de cet ensemble, les grandes agglomérations sont structurellement plus proches de Zurich (Berne surtout) que les autres, notamment argoviennes et soleuroises: un quadrilatere riche qui recèle en son centre des régions plus modestes.

\section{- Villes et agglomérations de l'Ostschweiz:}

Les villes et agglomérations de la Suisse orientale présentent de fortes différences avec le Triangle d'Or. Cet ensemble régional tendrait à avoir une répartition de ses communes nettement plus proche de celle qu'on observe en Argovie: un mélange de communes homogènes et de 


\section{Géographie de la force de travail de la Suisse urbaine, 1990}

( 805 unités spatiales et 46 caractéristiques)

Analyse en composantes principales, solution Varimax à 5 composantes, $62.9 \%$

\section{Composante 5}

Variance expliquée: $\quad 5.8 \%$

Variance cumulée : $\quad 62.9 \%$

\section{Structure}

Caractéristiques

Saturations +

Profil corrélatif des variables sur l'axe

Actifs féminins tertiaires au dom. $\quad 0.64$

Actifs féminins tertiaires au trav. $\quad 0.63$

Actifs féminins au trav. $\quad 0.48$

Actifs féminins primaires au trav. $\quad 0.44$

Actifs féminins primaires au dom. 0.43

Actifs secondaires au dom. $\quad 0.38$

Actifs féminins au dom. $\quad 0.36$

Employés au dom.

$-0.36$

Actifs tertiaires au dom. $\quad-0.44$

Saturations -

\section{Poids locaux}

Projection des unités spatiales sur l'axe

Unités spatiales

Ecarts-types

484. Zürich

1. Orselina (Locarno)

2. Scherzingen (Kreuzlingen)

3. Gempen (Basel)

4. Roveredo (Lugano)

5. Zielebach (Solothurn)

7. Echichens (Lausanne)

11. Le Locle

24. Regensberg (Zürich)

97. Genthod (Genève)

124. Grafenried (Bern)

239. Basel

285. Bern

324. Genève

350. Lausanne
4.17
2.56
2.46
2.44
2.38
2.33
2.14
1.70
1.10
0.97
0.54
0.40
0.25
0.17

684. Birsfelden (Basel)

$-0.95$

$-1.05$

697. Ittigen (Bern)

$-1.59$

756. St-Cergue (Genève)

$-1.94$

779. Etagnières (Lausanne)

787. Chiasso

$-2.32$

789. Oetwil / Limmat (Zürich)

801. Gnosca (Bellinzona)

$-2.37$

$-3.02$

802. Sagno (Chiasso-Mendrisio) $\quad-3.15$

803. Gorduno (Bellinzona) -3.18

804. Villarsel / Marly (Fribourg) $\quad-3.64$

805. Carabietta (Lugano) $\quad-4.77$

\section{Définition de la composante}

Un facteur féminin (emplois et résidence)

\section{IDENTIFICATION}

Le genre 


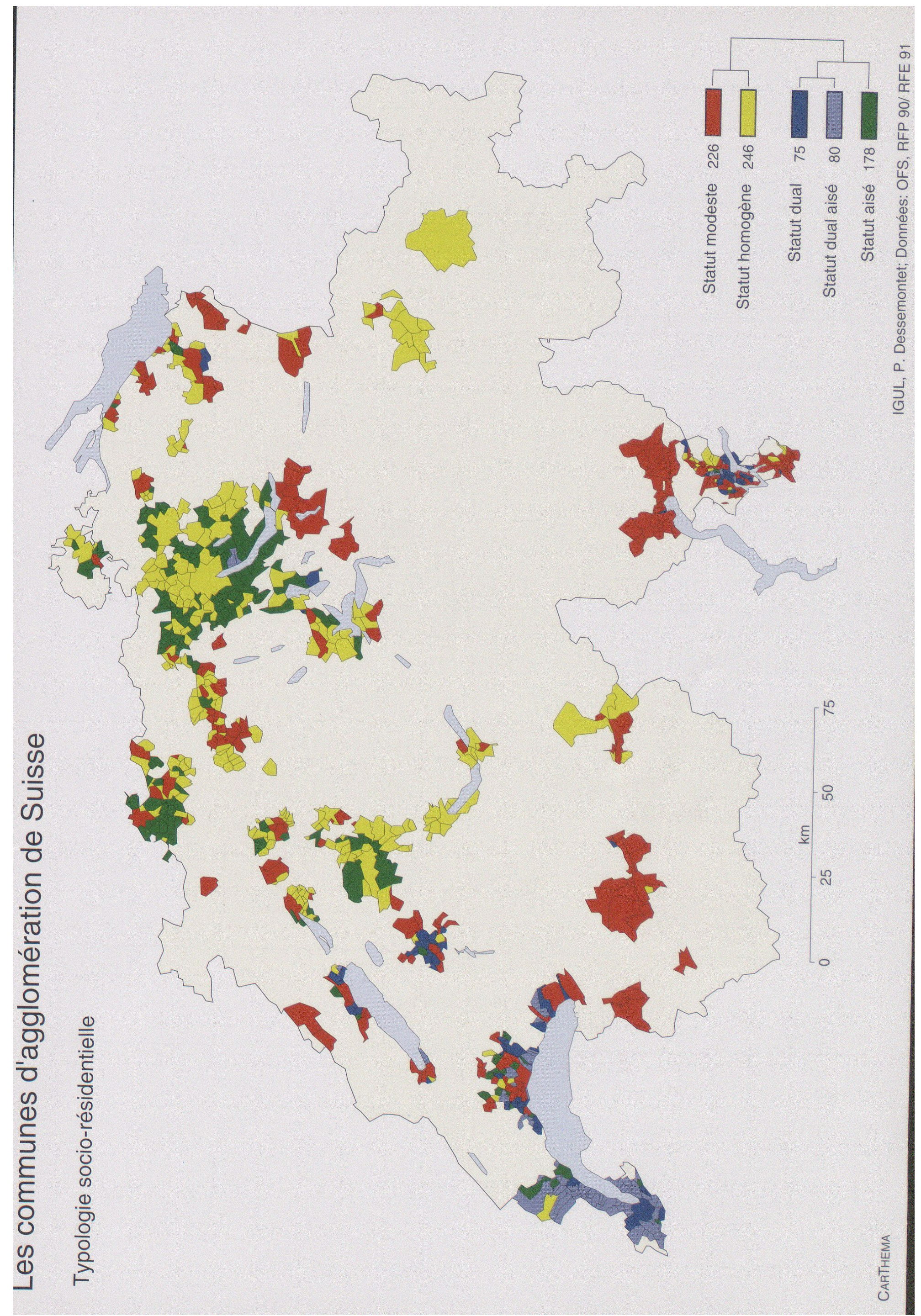


communes modestes, les secondes étant cependant plus nombreuses que les premières, à l'inverse de l'Argovie. Ce type concerne les agglomérations des cantons de St-Gall, de Thurgovie et de Schwyz. Une version plus modeste en fait du type précédent, ayant en commun avec ce dernier l'homogénéité de sa société sans en avoir la richesse. En miroir, quelques agglomérations de Suisse alémanique occidentale répondent également à cette définition.

- Villes et agglomérations de la Suisse latine de plaine:

La répartition des communes par type en Suisse latine (Genève exceptée) diffère complètement des deux types précédents: on a ici un mélange de communes duales et de communes modestes, en proportion à peu près égale, avec quelques communes homogènes, aisées et duales aisées. Le trait principal de ces agglomérations réside dans la part considérable qu'y occupent les classes dominées de la société, et le caractère dual de nombre de ces communes. Ce type concerne essentiellement les agglomérations latines de plaine, nommément Lausanne, Fribourg, Neuchâtel, Vevey-Montreux (soit les plus grandes agglomérations de Romandie après Genève), mais aussi Yverdon et, au Tessin, Lugano.

- Genève:

Cas spécial du type précédent, Genève s'en distingue par la combinaison quasi exclusive des communes duales et duales aisées. Genève est l'archétype de l'agglomération dont la société combine très riches et très pauvres. Une «corruption» probablement due au statut international de la ville, qui y attire nombre de personnes appartenant aux classes sociales dominantes, sans pour autant que la part de personnes de la classe servante ne baisse de manière significative. Autant la croissance zurichoise peut apparaître "orthogénétique», autant il semble que celle de Genève soit nettement "hétérogénétique» (VILLENEUVE 1981, RACINE 1994).

- Villes et agglomérations des montagnes et piémonts:

Dernier type que nous distinguons, les agglomérations de piemont ou de montagne sont essentiellement constituées de communes modestes: une société homogène et pauvre. Ce type concerne essentiellement le Valais (notamment romand) et le Tessin, mais on le trouve également en Suisse alémanique, notamment orientale (de Schwyz à Altstätten), et dans le Jura romand (La Chauxde-Fonds et Delémont).

\section{Quelques leçons additionnelles d'une classification économique et fonctionnelle}

En regard de cette classification "sociale» notre analyse permettrait d'en envisager une seconde, économique et fonctionnelle, combinant les facteurs 2 et 4 de l'analyse, à savoir celui qui opposait centre et périphérie (en termes de proportion d'agriculture surtout) et celui qui opposait spécialisations secondaires et tertiaires. Sans en reprendre ici le détail, notons simplement qu'elle met en évidence deux types de périphéries et deux types de centres, communes d'emplois essentiellement non agricoles, se différenciant cependant en fonction de la part qu'y tiennent l'industrie et le tertiaire.
L'examen de la carte des répartitions des communes selon cette typologie en quatre classes permet certes de tirer de nombreux enseignements. Le premier est que contrairement à la carte des répartitions socio-économiques, les différences entre grandes régions apparaissent peu, et qu'elles sont bien moindres en tous les cas que celles observées au sein des agglomérations. Ce qui revient à dire que structurellement, les agglomérations du pays se ressemblent entre elles - celles de taille analogue du moins -, par la manière dont, en fonction de leurs activités respectives, se divisent les communes qui les forment. Il apparaît en effet que la partition entre centre et périphérie diffère fortement selon la taille de l'agglomération; de même, les effets de métropolisation autour des grandes conurbations helvétiques ont une influence certaine sur le développement des agglomérations de taille moyenne. Deux phénomènes, deux effets de taille en fait, qui se marquent sur l'opposition centre-périphérie: outre l'effet de masse, l'effet de métropolisation qui en est un dérivé. Une analyse plus poussée aurait pu distinguer, dans la ligne de cet autre travail de l'équipe de l'IREC, l'effet vertical, lié au pouvoir, et l'effet horizontal, lié à l'expansion spatiale (JOYE, BUSSET et SCHULER 1992).

On notera cependant que le premier caractère remarquable de l'opposition entre communes centrales et communes périphériques réside dans le fait que plus l'agglomération est importante, plus la part des communes centrales au sein de l'agglomération l'est également. Ce qui signifie d'une part que la centralité des grandes agglomérations est plus forte, en Suisse, que celles de petites (un truisme!), mais encore que cette centralité tend à se diffuser à l'ensemble de l'agglomération en fonction de sa taille.

C'est évidemment dans celle de Zurich que cet effet de diffusion est le plus manifeste: ici, les communes centrales occupent l'essentiel de l'agglomération. Outre quelques îlots, seules quelques communes, à l'ouest et au nord, échappent à cette catégorie. Zurich est la seule agglomération suisse (en plus d'être de loin la plus importante) dont le caractère de centralité des communes de l'agglomération soit aussi poussé. Il l'est déjà nettement moins dans les quatre autres grandes villes suisses, qui toutes comportent une part assez notable de communes périphériques, même si, là encore, elles sont en minorité. Il arrive en revanche qu'au sein de ces agglomérations apparaissent des centres secondaires, ce qui n'est pas le cas à Zurich où l'essentiel de l'agglomération est central. En termes de surface, on constate également que Bâle, Genève, Berne et Lausanne ont une zone centrale qui pour être nettement plus étendue que dans les autres agglomérations du pays, sont nettement plus petites que celle de Zurich. Autant dire que si on évoque si souvent en Suisse "les cinq grandes», il serait plus conforme à la réalité de parler de Zurich et des quatre autres.

Au sein des agglomérations moyennes, de grandes disparités apparaissent. Il y a d'une part celles qui comportent une grande zone centrale dominante: Lucerne, Lugano, Zoug, les agglomérations argoviennes et la majorité de celles du pied du Jura, de l'autre, celles qui comportent 
un centre encore essentiellement cantonné au seul noyau de l'agglomération, comme Winterthour, St-Gall, Fribourg, et l'essentiel des petites agglomérations. Notons en revanche qu'il n'est pas rare de ne recenser qu'une ou deux communes centrales dans les petites agglomérations dont la majorité des communes sont restées des communes périphériques. C'est le cas en Valais, en Suisse orientale, en Suisse centrale.

Il reste qu'une dichotomie apparaît nettement entre les agglomérations moyennes. Les agglomérations moyennes à grande zone centrale se concentrent autour de Zurich ou dans son prolongement: ainsi des argoviennes, de Zoug, de Lucerne, de Schaffhouse. De même, toutes les agglomérations proches de Zurich montrent une centralité très étendue, indépendamment de leur taille: ainsi de Wetzikon-Pfäffikon, de Rapperswil, de Baden. A l'inverse, ce phénomène de centralité étendue n'apparaît que peu ailleurs dans le pays: essentiellement dans le pied du Jura (à la longue histoire urbaine, ce qui explique peut-être cette situation), presque nulle part ailleurs. Un phénomène qui se relie sans doute à la présence de Zurich et à la constitution autour de cette dernière de la métropole du Triangle d'Or, Zurich possédant de multiples relais urbains qui lui sont fonctionnellement similaires. Ailleurs, aucun phénomène similaire ne semble avoir pris place, ce qui expliquerait le caractère, encore essentiel, de "petite ville» que montrent les agglomérations moyennes.

Deux phénomènes permettent donc d'expliquer l'étendue des zones centrales dans les agglomérations suisses. Le premier est l'effet de taille: plus une agglomération est grande, plus la part de la zone centrale est développée par rapport au reste de l'agglomération; pour les agglomérations moyennes, un second facteur interfère avec le premier: la proximité d'avec une grande agglomération joue également un rôle: à taille égale, une agglomération moyenne aura une zone centrale plus développée si elle entretient des liens (proximaux ou fonctionnels) avec une grande agglomération, c'est-à-dire si elle est partie prenante d'un processus de métropolisation.

Au total, la Suisse urbaine et métropolitaine n'échappe pas, n'échappe plus, au modèle dualiste. L'analyse factorielle l'a confirmé à bien des échelles, de l'échelle nationale à l'échelle intramétropolitaine. D'autres études le confirmeront au niveau intracommunal: ce qui est vrai des individus tend à l'être également des territoires qu'ils occupent préférentiellement: d'un côté ceux qui sont «in», intégrés dans le monde du travail, bénéficiant d'un emploi qualifié, branché sur les réseaux sociaux et jouissant d'un niveau de vie confortable; de l'autre ceux qui sont «out», qui n'ont pas accès à l'emploi, qui subissent la précarité économique et l'isolement social. Et c'est bien dans la société urbaine que se manifeste le plus nettement aujourd'hui la "fabrique des exclus».

Sans doute, les métropoles sont-elles à la fois le moteur et le miroir de nos sociétés. A côté de leur évident potentiel, tant culturel qu'économique et social, la pauvreté et la ségrégation spatiale sont aussi des caractéristiques des grandes agglomérations. Le manque d'infrastructures et de services, mais aussi la dégradation environnementale qui en caractérisent d'autres, les difficultés d'ajustement local aux mutations technologiques: autant de problèmes au cœur du débat sur le développement urbain dans lequel entrent à leur tour, les villes, si volontiers qualifiées de "rescapées», de l'Helvétie. Un débat qui ne saurait évacuer le problème de la recherche de niveaux géographiques mieux adaptés à la gestion de ces réalités nouvelles.

\section{Vers une nouvelle "gouvernance» urbaine?}

Alors même que la ville est en passe de devenir, comme elle le fût au Moyen Age notamment, la structure essentielle de la société de l'Europe occidentale au siècle prochain, la ville suisse reste enfermée dans une structure politique qui ne lui confère guère de pouvoir. Et les problèmes des villes ne sont pas considérés comme les problèmes du pays tout entier, mais comme ceux des communes urbaines, tout se passant comme si le pays vivait encore dans la peur de voir les villes, leur culture et leur politique se répandre sur leur arrière-pays parallèlement à l'évolution démographique et économique. Certes, on connaît la réitération répétée des Suisses voulant que leur attachement consensuel à leur particularisme institutionnel soit basé sur la supériorité morale, politique et sociale de la Gemeinschaft (le lien social communautaire) sur la Gesellschaft (le lien plus abstrait de type social). Il n'en demeure pas moins que le hiatus entre la réalité urbaine et le registre onirique dominant dans les périphéries même immédiates des centres est si grand qu'encore actuellement les enquêtes montrent non seulement qu'entre communes du centre-ville et communes urbaines périphériques, l'opposition est souvent aussi forte qu'entre ce que l'on appelle encore de manière trop schématique "ville» et "campagne» mais encore que ne se reconnaissent réellement comme «urbains» que les habitants des centre-villes, héritage sans doute des représentations spécifiques de la ville dans ce pays, marquées par la persistance, dans ce pays féru pourtant d'«aménagement du territoire», des idéologies ruralisantes du XIXe et XXe siècle, celle du "village suisse» en particulier, ainsi que de l'importance des attitudes anti-urbaines dans la culture politique, celles-ci ayant longtemps différé la prise de conscience de l'intérêt des investigations spécifiques au champ urbain (WALTER 1994; SALOMON 1996).

L'heure n'est-elle pas venue d'imaginer les moyens de lutter contre des habitudes laissant s'opposer des communes qui se comprennent elles-mêmes si volontiers comme "différentes» en laissant jouer, sous prétexte d'identité ou de risque de déficit démocratique, les intérêts et les égoïsmes locaux alors que chacun sait aujourd'hui, et cette étude le confirme, que l'opposition n'est plus simplement entre le centre et la périphérie, mais, 
parmi les communes urbaines périphériques ellesmêmes, entre les communes les plus riches (celles des "côtes dorées" par exemple) et les plus pauvres (celles des grands ensembles ou des habitats les moins bien situés, en bordure des autoroutes ou des plus grands axes de circulation notamment (CUDET 1996).

Etat fédératif, la Suisse répartit l'essentiel des compétences et du pouvoir politique entre la Confédération et les cantons. Dans cette dualité, les villes comme telles sont complètement absentes et non reconnues au plan institutionnel. Au sein même de la circonscription administrative qu'est également la structure cantonale, les villes sont le plus souvent minoritaires et leurs intérêts spécifiques passent régulièrement au second plan par rapport à l'intérêt des communes rurales beaucoup plus nombreuses. Et il n'est pas rare que la représentation politique, d'une manière générale en Suisse, reste majoritairement d'inspiration et de vision plutôt rurale alors que près des trois quarts de la population vivent dans des villes ou des agglomérations ou y gagnent leur vie. Alors que compte tenu de la rapidité des processus de métropolisation en cours, les choix stratégiques pouvant préserver de façon active la cohésion sociale et la qualité des espaces urbains sont difficiles à élaborer, impossibles à implanter dans le cadre d'un développement urbain abandonné aux seules exigences de la compétitivité économique ou des logiques privées, sectorielles ou administratives d'appropriation de l'espace, le cadre communal est inadéquat, et les cantons font plus souvent qu'à leur tour barrage à une authentique politique urbaine conçue à l'échelle nationale. De là la proposition actuellement soumise par l'union des villes suisses dans le cadre de la révision totale de la Constitution fédérale, d'un nouvel article constitutionnel qui donnerait enfin un statut institutionnel aux villes et à leurs régions. Une proposition intéressante mais notoirement insuffisante dans la mesure où elle ne vise en fait que les problèmes d'ordre financier.

C'est pourquoi de nombreuses propositions annexes voient actuellement le jour visant à des transformations institutionnelles susceptibles de permettre de mieux prendre en compte les populations concernées, en imaginant en particulier, comme cela vient d'être fait pour Fribourg et en cours de consultation pour Lausanne, la création, à travers une nouvelle loi sur les agglomérations, d'entités cohérentes correspondant à l'espace vécu et pratiqué centrées sur les chefs-lieux, certes, mais entourée des deux cercles formés par la zone de débordement du noyau originel d'une part, mais aussi par une région qui tout en restant à dominante rurale, accueille les nombreux pendulaires vivant en symbiose avec le centre et le premier cercle, définissant ainsi, objectivement, une communauté d'intérêts dans un ensemble solidaire, et structuré de manière cohérente et doté d'un statut légitime et nouveau dans l'ordre juridique du pays, et donc des pouvoirs démocratiques adéquats, alors même que force est de constater le désintérêt croissant de ces deux cercles pour des problèmes communs que la ville-centre résout toute seule.
Mais une telle problématique, qui paraît si évidente à tous les géographes, n'en est en Suisse, un pays où sévit particulièrement l'égoïsme socio-spatial, qu'aux premiers balbutiements, seule Fribourg s'étant réellement lancée dans cette démarche visant à donner aux citoyens de deux communes limitrophes la possibilité de déclencher, sans aucun moyen de retour, un processus de constitution d'une telle agglomération. On est donc encore loin des étapes suivantes, la mise en réseau des entités urbaines ainsi constituées, elles-mêmes en réseau avec des entités urbaines secondaires, la reconnaissance institutionnelle enfin de ce vaste maillage.

Les villes, les agglomérations urbaines et métropolitaines doivent être gérées et gouvernées. Le contexte d'incertitude qui tend à imposer de nouvelles modalités de gestion collective du changement et à susciter de nouvelles interrogations sur l'avenir de nos sociètés, réclame simultanément la prise en compte de l'action et des aspirations du réseaux d'acteurs au niveau local, suivant, par exemple, les logiques du partenariat évoquées tant par F. ASCHER (1995) que par P. LE GALES (1995) et une authentique insertion dans les logiques interrégionales et internationales. En d'autres termes, l'intégration de nouvelles échelles pertinentes de l'analyse et de l'action. Les nouveaux cadres spatiaux de la vie urbaine doivent être reconnus, institutionnalisant le dialogue entre les autorités fédérales et les villes, en particulier sur les questions de politique sociale. C'est d'ailleurs l'objet d'une «initiative pour les villes" réclamée depuis longtemps mais qui peine encore à faire entendre sa voix dans un pays trop marqué par l'idéologie politique du consensus obligé et des petits pas politiques alors même, (l'un de ces nombreux paradoxes helvétiques parmi tant d'autres) (RACINE et RAFFESTIN 1996), qu'il n'hésite souvent pas, quand il s'agit de faire des bonds en matière de restructuration et de globalisation économique. Reste à savoir si la tentation qui se fait de plus en plus forte pour la gouvernance des systèmes urbains, envisagée à chacune des échelles pertinentes, est compatible avec des processus qui apparaissent de plus en plus nettement comme des processus d'auto-organisation (PUMAIN 1996).

\section{Bibliographie}

ASCHER, F. (1995): Métapolis ou l'avenir des villes. Paris. AYDALOT, Ph. (1985): Economie régionale et urbaine, Paris, Economica.

CUDET, A. (1996): Ville réelle et ville institutionnelle: proposition de réorientation pour l'activité du mouvement “Un projet pour les villes". Genève.

CUNHA, A. (1993): Développement territorial, régions et centralité urbaine, Lausanne. Thèse HEC, Université de Lausanne. 
CUNHA, A. (1994): L'avenir de la Suisse urbain: changement technologique, métropoles informationnelles et développement territorial. Dans: Geographica Helvetica, no2, pp. 53-62.

CUNHA, A. (1995): Territorialisation, métropolisation et globalisation, Métamorphoses de la centralité, reconfigurations du territoire. Dans: DISP, 122, juillet, pp. 3-13.

CUNHA, A. (1996 a): Métropolisation, processus d'exclusion et développement durable. Dans: JACCOUD, SCHULER, BASSAND (1996): Raisons et déraisons de la ville: approches du champ urbain. Lausanne, PPUR, pp. 21-44.

CUNHA, A. (1996 b): Mobilité spatiale et centralité urbaine: qualifications, statuts socio-professionnels et migrations sélectives. IREC, EPFL.

CUNHA A., RACINE, J. B. (1986): Services Agencies in Swiss Regional Dynamics, From Cumulative Growth to Polycentric Decentralization, in: J. G. Borchert, L. S. Bourne et R. Sinclair, éd. Urban Systems in Transition, Nederlandse Geografische Studies, 16, pp. 99-111.

CUNHA A., RACINE, J.B. (1992): Changements structurels et évolution du système urbain helvétique: tertiairisation sélective et industrialisation diffuse. Dans: Canadian Journal of Regional Science, Vol.XV. 3. pp. 377-402.

DANIELS, P. (1993): Activités tertiaires et dynamique des métropoles: comparaisons internationales, dans: DATAR, Métropoles en déséquilibre? Paris, Economica, pp. 397-430.

LE GALĖS, P. (1995): Du gouvernement des villes à la gouvernance urbaine. Dans: Revue des sciences politiques, mai.

PIVETEAU, J.L. (1990): Du Moyen Age à nos jours: le couplage industrie-ville. Dans: Racine, J. B., et Raffestin, C. (1990), Nouvelle Géographie de la Suisse et des Suisses, Lausanne, Payot, tome I, pp.121-144.

PUMAIN, D. (1996): Le temps et les villes. Dans: UKPIK (Cahiers de l'Institut de géographie de l'Université de Fribourg) No 11, pp.17-32.
RACINE, J.B. (1994): Développement urbain métropolitain et crise de la ville: un monde en transition. Dans: Revue suisse d'Economie politique et de Statistique, 128 (3), pp. 241-260.

RACINE, J.B. (1994): Langues et identités territoriales en Suisse: les leçons géographiques d'un vote historique ou la Suisse à l'heure du rideau de rösti. Dans: Annales de Géographie, no 576, p.152-169.

RACINE, J. B. (1994): La Suisse urbaine à l'orée du 3e millénaire: risques et défis d'un changement non programmé. Dans: Geographica Helvetica, vol. 49, (2) p. 49-52.

RACINE, J.B., CUNHA, A. (1991): Invariance and Structural Changes in the Swiss Urban System: The Leading Role of Tertiary Activities. In: Larry S.Bourne et al., (ed.): The Changing Geography of Urban Systems, Perspectives on the Developed and Developing Worlds, IGU Universitad de Navarra: pp.145-172.

RACINE, J.B., RAFFESTIN, C. (1990): Nouvelle Géographie de la Suisse et des Suisses. Lausanne, Payot, 2 tomes.

RACINE, J.B., RAFFESTIN, C. (1996): La Suisse à I'heure des paradoxes, ch. 13. Dans: Géographie Universelle R. Brunet (ed.) Europe du Nord, Europe Médiane, Paris, Belin-Reclus, pp. 400-419.

ROSSI, A. (1983): La décentralisation urbaine en Suisse. Lausanne, Presses polytechniques romandes.

ROSSI, A. (1996): Concurrence territoriale et réseaux urbains, L'armature urbaine de la Suisse en transition. Zurich, V/D/F, PNR Ville et transport.

ROSSI, A., FILIPPINI, M. (1994): Mobility of Firms and Territorial Competition: the Zurich Metropolitain Area Case. ORL, EPFZ.

SCHULER, M. (1994): Les niveaux géographiques de la Suisse. Berne, OFS.

WALTER, F. (1994): La Suisse urbaine, 1750-1950. Genève, Zoé. 